\title{
Chronic Presence of Blood Circulating Anti-NMDAR1 Autoantibodies Impairs Cognitive Function in Mice
}

\author{
William Yue ${ }^{1}$, Sorana Caldwell ${ }^{1,2,3}$, Victoria Risbrough ${ }^{1,2,4}$, Susan Powell ${ }^{1,2,3}$, Xianjin \\ Zhou ${ }^{1,2,3 *}$ \\ ${ }^{1}$ Department of Psychiatry \\ University of California San Diego \\ La Jolla, California, United States of America \\ ${ }^{2}$ VA Research Service \\ ${ }^{3}$ VA Mental IIIness Research and Clinical Core \\ ${ }^{4}$ VA Center of Excellence for Stress and Mental Health \\ VA San Diego Healthcare System \\ San Diego, California, United States of America
}

* To whom correspondence should be addressed: Department of Psychiatry, University of California San Diego, La Jolla, USA. Tel: 858-822-3709; Fax: 858-5345527. (Email: xzhou@ucsd.edu)

Short title: Anti-NMDAR1 autoantibodies in mice 


\section{Abstract}

High titers of anti-NMDAR1 autoantibodies in brain cause anti-NMDAR1 encephalitis that displays psychiatric symptoms of schizophrenia and/or other psychiatric disorders in addition to neurological symptoms. Low titers of anti-NMDAR1 autoantibodies are reported in the blood of a subset of the general human population and psychiatric patients. Since $\sim 0.1-0.2 \%$ of blood circulating antibodies cross the blood-brain barriers and antibodies can persist for months and years in human blood, it is important to investigate whether chronic presence of these blood circulating antiNMDAR1 autoantibodies may impair human cognitive functions and contribute to the development of psychiatric symptoms. Here, we generated mice carrying low titers of anti-NMDAR1 autoantibodies in blood against a single antigenic epitope of mouse NMDAR1. Mice carrying the anti-NMDAR1 autoantibodies are healthy and display no differences in locomotion, sensorimotor gating, and contextual memory compared to controls. Chronic presence of the blood circulating anti-NMDAR1 autoantibodies, however, is sufficient to specifically impair T-maze spontaneous alternation in the integrity of blood-brain barriers across all 3 independent mouse cohorts, indicating a robust cognitive deficit in spatial working memory and/or novelty detection. Our studies implicate that chronic presence of low titers of blood circulating anti-NMDAR1 autoantibodies may impair cognitive functions in both the general healthy human population and psychiatric patients.

Key Words: Anti-NMDAR1 autoantibodies, antigenic epitope, cognitive function, mouse model, chronic effect, active immunization 


\section{Introduction}

$\mathrm{N}$-methyl-D-aspartate receptor (NMDAR) in brain is essential for learning and memory as well as other cognitive functions. Pharmacological studies demonstrated that NMDAR antagonists cause schizophrenia-like symptoms in human (Javitt and Zukin, 1991). Recent human genetic studies further validated the central role of NMDAR functions in the development of schizophrenia (Tarjinder Singh, 2020; The Schizophrenia Working Group of the Psychiatric Genomics Consortium, 2020). In addition to genetic mutations, NMDAR functions can also be impaired by physiological/environmental risk factors. High titers of anti-NMDAR1 autoantibodies in human brain cause anti-NMDAR1 encephalitis that exhibits psychosis, memory loss, and other prominent psychiatric symptoms in addition to neurological symptoms (Dalmau et al., 2008; Dalmau et al., 2011). In fact, anti-NMDAR1 encephalitis is often misdiagnosed as schizophrenia, further supporting impaired NMDAR functions in the pathogenesis of schizophrenia and other psychiatric disorders.

Low titers of anti-NMDAR1 autoantibodies are commonly detected in human blood. Several studies reported that $\sim 5-10 \%$ of the human population, regardless of healthy persons or psychiatric patients, carries low titers of anti-NMDAR1 autoantibodies in their blood (Hammer et al., 2014; Schou et al., 2016; Steiner et al., 2013). These anti-NMDAR1 autoantibodies inhibit NMDAR functions in both in vitro human neurons (Castillo-Gomez et al., 2016) and in vivo mouse models (Hammer et al., 2014; Planaguma et al., 2015). Although peripheral circulating antibodies are largely blocked from entering brain parenchyma by the blood-brain barriers (BBB), $\sim 0.1-0.2 \%$ of blood circulating antibodies can cross the BBB into brain tissue in healthy rodents 
and humans regardless of antibody specificities (Fossan, 1977; St-Amour et al., 2013; Wang et al., 2018). This raises a key question as to whether chronic presence of low titers of anti-NMDAR1 autoantibodies in human blood may impair human cognitive functions and contribute to the pathogenesis of schizophrenia and other psychiatric disorders. Mouse models carrying anti-NMDAR1 autoantibodies have been generated via active immunization of NMDAR1 holoreceptor (Jones et al., 2019) or mixtures of long NMDAR1 peptides (Pan et al., 2018); but different behavioral phenotypes were reported. Neither the human studies nor the mouse studies have so far however characterized anti-NMDAR1 autoantibodies at the level of individual antigenic epitopes. Here, we generated mice chronically carrying low titers of blood anti-NMDAR1 autoantibodies against a single NMDAR1 antigenic epitope. Since disrupted NMDAR neurotransmission causes hyperlocomotion, impaired sensorimotor gating, and deficient memory, we characterized these behavioral phenotypes in the mice carrying the antiNMDAR1 autoantibodies.

\section{Materials and Methods}

Mouse strain

C57BL/6J mice were purchased from Jackson Labs (Bar Harbor, ME) at 8-week-old and housed in a climate-controlled animal colony with a reversed day/night cycle. Food (Harlan Teklab, Madison, WI) and water were available ad libitum, except during behavioral testing. Active immunization was conducted after a week acclimation. A few microliters of blood were taken from mouse tail vein at multiple time-points (WK3, WK4, WK5, WK7, WK9, WK13, WK24) after immunization to detect production of anti- 
NMDAR1 autoantibodies. Behavioral testing began when mice were 24 weeks postimmunization or stated otherwise. All testing procedures were approved by UCSD or local VA Animal Care and Use Committee prior to the onset of the experiments. Mice were maintained in American Association for Accreditation of Laboratory Animal Care approved animal facilities at the local Veteran's Administration Hospital or UCSD campus. These facilities meet all Federal and State requirements for animal care.

Active immunization

Peptides P1 and P2 from mouse NMDAR1 were synthesized by Biomatik. The P1 (KLVQVGIYNGTHVIPNDRKI) and the P2 (TIHQEPFVYVKPTMSDGTCK) peptides are located in the amino terminal domain and the ligand binding domain of mouse NMDAR1 protein, respectively. Mycobacterium tuberculosis, H37 RA (Difco) and Incomplete Freund's Adjuvant (Bacto) were purchased. The peptide immunization was conducted as described for EAE mouse model (McCarthy et al., 2012). In brief, the P2 peptide was dissolved in PBS at a concentration of $4 \mathrm{mg} / \mathrm{ml}$. An equal volume of the P2 solution is completely mixed with complete Freund's adjuvant (containing $4 \mathrm{mg} / \mathrm{ml}$ desiccated M. Tuberculosis, H37 RA in Incomplete Freund's Adjuvant) to generate a thick emulsion. Mice were injected with $100 \mathrm{ul}$ of emulsion subcutaneously divided equally at 3 sites on mouse flank. For the injection of the control mice, the same emulsion was generated but without the P2 peptide. Blood was taken from mouse tail vein for detection of anti-NMDAR1 autoantibodies.

\section{Immunohistochemistry}

Immunohistochemistry (IHC) was conducted as previously described (Ji et al., 2013;

Kim et al., 2012) to evaluate the production of anti-NMDAR1 autoantibodies against the 
P2 peptide and their semi-quantifications. Mouse blood was diluted at 1:200 with antibody diluent solution (DAKO, S080983-2) as the primary antibodies for IHC on wildtype mouse brain paraffin sections. Mouse anti-NMDAR1 monoclonal antibody (BD, cat. 556308) was diluted at 1:40,000 as a positive control. ImmPRESS peroxidasemicropolymer conjugated horse anti-mouse IgG (Vector Labs, MP-7402) was used as the secondary antibody. Chromogenic reaction was conducted with ImmPACT NovaRED Peroxidase Substrate (Vector Labs, SK-4805). Slides were mounted with Cytoseal 60 mounting medium (Richard-Allan Scientific, 8310-16). Optical intensities were measured with Image $\mathrm{J}$, and differential intensities between hippocampal CA1 st oriens and corpus callosum were used as the surrogates for the levels of the antiNMDAR1 autoantibodies. The anti-NMDAR1 autoantibodies persisted more than 1 year in mouse blood. Mouse brains were fixed $\sim 10$ months after immunization. Immunohistochemical analysis was conducted to investigate expression of NMDAR1, GluR1 and GFAP in the hippocampus, cortex, and striatum. Rabbit anti-NMDAR1 (Abcam, Ab17345), GluR1(Abcam, Ab31232), GFAP (Abcam, ab68428) antibodies were diluted at 1:5,000,1:20,000,1:10,000, respectively, for immunohistochemical staining.

\section{Peptide blocking experiments}

Peptide blocking $\mathrm{IHC}$ experiments were conducted to evaluate the specificity of the staining by the autoantibodies against the NMDAR1 P2 antigenic peptide. Bovine serum albumin (BSA), the P1 peptide, the P2 peptide were pre-incubated with the diluted mouse blood containing the anti-NMDAR1 autoantibodies at the concentration of $25 \mathrm{ng} / \mathrm{ul}$ for 1 hour at room temperature, respectively. After pre-incubation, these 
mixtures were used as the primary antibodies for IHC staining on paraffin brain sections of wildtype mice.

Cell-based assay

Immunofluorescence analysis of mouse anti-NMDAR1 autoantibodies were conducted using BIOCHIP (Euroimmun, FB 112d-1005-51) with a positive control of human antiNMDAR1 autoantibody (Euroimmun, CA 112d-0101). Mouse anti-NMDAR1 monoclonal antibody (BD, cat. 556308), diluted at 1:10,000 with antibody diluent solution (DAKO, S080983-2), was also included as a positive control for the cell-based assay. Serum from mice immunized with the NMDAR1 P2 peptide was diluted at 1:10 with DAKO antibody diluent solution. Goat anti-Human IgG (H+L) Fluorescein (Vector Lab, Fl-3000) and goat anti-mouse IgG $(\mathrm{H}+\mathrm{L})$ Alexa Fluor 568 (Invitrogen, A11004) were diluted at $1: 1,000$ as the secondary antibodies for immunofluorescence staining. The cell-based assay was conducted as recommended by the Euroimmun manufacturer.

Flurorescence staining was examined using microscope EVOS FL (ThermoFisher, Scientific).

One-Step quick assay

The NMDAR1 P2 peptide or NMDAR1 ligand binding domain was fused with GFP tagged with $6 \mathrm{His}$ for the One-Step quick assay to screen immunized mice for the generation of anti-NMDAR1 autoantibodies (Zhou, 2021). The fusion genes were synthesized and cloned into pET-21d vector and transformed into BL21 (DE3) pLysS E coli cells (EMD, 70236-3). Over-expression and purification of NMDAR1-GFP fusion proteins and subsequent One-Step assay were conducted as described (Zhou, 2021). 


\section{Video-Tracker}

All mouse behavioral tests were conducted during their dark cycle with lights on for the entire duration of the test. Locomotor activity was measured using a Video-Tracker (VT) system in open field for $60 \mathrm{~min}$ as previously described (Ji et al., 2013). Briefly, mice were first acclimated to the testing room for 60 min before placed into white plastic enclosures $\left(41 \times 41 \times 34 \mathrm{~cm}^{3}\right)$ surrounded by an opaque plastic curtain. A video camera, mounted $158 \mathrm{~cm}$ above the enclosures, generated the signal for the Ethovision 3.0 (Noldus; Leesburg, VA) to record distance moved and time/entries in specified zones.

\section{Behavioral pattern monitor}

The mouse Behavioral Pattern Monitor (mouse BPM) was used to record exploratory and investigatory behavior according to our previously published methods (Halberstadt et al., 2009; Risbrough et al., 2006). Each mouse BPM chamber (San Diego Instruments, San Diego, CA) is a transparent Plexiglas box with an opaque $30 \times 60 \mathrm{~cm}$ floor, enclosed in a ventilated isolation box. The position of the mouse in $x, y$ coordinates is recorded by a grid of $12 \times 24$ infrared photobeams located $1 \mathrm{~cm}$ above the floor. A second row of 16 photobeams (parallel to the long axis of the chamber, located $2.5 \mathrm{~cm}$ above the floor) is used to detect rearing behavior. Holepoking behavior is detected by 11, 1.4-cm holes that are situated in the walls ( 3 holes in each long wall, 2 holes in each short wall) and the floor (3 holes); each hole is equipped with an infrared photobeam. Photobeam status is sampled every $55 \mathrm{~ms}$ and raw beam breaks are transformed into $(x, y, t$, event) ASCII data files composed of the $(x, y)$ location of the mouse in the chamber with a resolution of $1.25 \mathrm{~cm}$, the duration of each event (t) and whether a 
holepoke or rearing occurred (event). The measures assessed were distance traveled (a measure of locomotor activity), total rearings and total holepokes (measures of investigatory behavior). Mice were acclimated to the testing room for 60 min prior to a 60 min session in the BPM. Testing room was dimly illuminated with a red light and chambers were kept dark.

\section{T-maze}

Spatial working memory and/or novelty detection was tested using the T-maze. The Tmaze apparatus is constructed of black Plexiglas (main stem is $45 \mathrm{~cm}$ long, $10 \mathrm{~cm}$ wide, $24 \mathrm{~cm}$ high; each side arm is $35 \mathrm{~cm}$ long, $10 \mathrm{~cm}$ wide, $24 \mathrm{~cm}$ high). Horizontal sliding doors separate the side arms from the stem. A start box, $8 \mathrm{~cm}$ in length, on the main stem is also separated by a horizontal sliding door. Testing was conducted under dim red light and white noise by an experimenter blind to group status. On the first day, mice were brought to the testing room for $60 \mathrm{~min}$ and handled for $5 \mathrm{~min}$ each. On the following day, mice were acclimated to the testing room for $60 \mathrm{~min}$ and placed in the Tmaze to explore the maze for $5 \mathrm{~min}$. On the third day, mice were again acclimated to the testing room for 60 min prior to spontaneous alternation testing. Each mouse was tested in a session of 8 successive free-choice trials. At the beginning of each trial, the mouse is placed in the start chamber for $30 \mathrm{sec}$. When the door is opened, the mouse is allowed to freely explore the maze. After the mouse chooses an arm, the door is closed and the mouse is kept in the arm for $30 \mathrm{sec}$. The chosen arm and the latency to choose the arm were recorded. Subsequent trials are run in the same manner with the mouse confined to both the start chamber and the arm for $30 \mathrm{sec}$ on each trial. Mice performed a total of 8 trials for a total of 7 possible alternations. 


\section{Prepulse inhibition}

Mouse startle reactivity and prepulse inhibition (PPI) were measured with startle chambers (SR-LAB, San Diego Instruments, San Diego, CA) as described (Ji et al., 2013).

\section{Fear conditioning}

Fear conditioning experiment was conducted as described in previous studies (Gresack et al., 2010; Toth et al., 2014) using automated fear conditioning chambers. Four Med Associates Inc. fear conditioning chambers were used (St. Albans, Vermont). Freezing was determined using video analysis software (Video Freeze, Med Associates Inc.). Footshocks were delivered through the 36 stainless steel rods located on the floor of the chamber. On Day 1 fear acquisition, after a 60-min habituation period in an adjacent room, mice were placed in the conditioning chamber. After an acclimation period (2 min), mice were presented with a tone (CS: $75 \mathrm{~dB}, 4 \mathrm{kHz}$ ) for $20 \mathrm{sec}$ that co-terminated with a foot shock (US: $1 \mathrm{sec}, 0.5 \mathrm{~mA}$ ). A total of 5 tone-shock pairings were presented with an inter-trial interval of $40 \mathrm{sec}$. Freezing was measured during tone presentations and for 40 sec after shock. Mice were placed back in their home cage 40 sec after the final shock. The chambers were cleaned with water after each session. On Day 2, mice were re-exposed to the conditioning chamber to assess context memory. The habituation period and features of the chamber were identical to those used during conditioning. Mice were then placed in the chambers for $16 \mathrm{~min}$ and tested for freezing during which time no shocks or tones were presented and freezing was scored. On Day 3, mice were tested for cued fear and fear extinction. The context of the chambers was altered across several dimensions (tactile, odor, visual) for this test in order to minimize 
generalization from the conditioning context. After a 5 min acclimation period, during which time no tones were presented ("pre-tone"), 32 CS tones were presented to test cued fear and cued fear extinction. On Day 4, recall of fear extinction was examined. After a 5 min acclimation period without CS tone, 12 CS tones were presented to test recall of fear extinction.

\section{Statistical analysis}

R programming was used for statistical analyses of effect size, power, repeated measures analysis of variance (ANOVA) with anti-NMDAR1 autoantibody and sex as between-subjects factor, block and prepulse intensity as within-subjects factors were performed on the \%PPI data and total distance traveled. For fear conditioning, ANOVA was conducted with anti-NMDAR1 autoantibody and sex as between-subjects factor and CS or time block as a within-subject factor for freezing percentages. Post hoc analyses were carried out using Newman-Keuls or Tukey's test. Alpha level was set to 0.05 .

\section{Results}

To generate mice carrying anti-NMDAR1 autoantibodies against a single antigenic epitope, we immunized mice with a small synthetic peptide of 20 amino acid residues that can only accommodate a single antigenic epitope for $\mathrm{MHC}$ class II molecules (binding antigen peptides ranging from 15-24 amino acid residues). Two NMDAR1 peptides P1 and P2, each 20 amino acids long, were selected from the amino terminal domain (ATD) and the ligand binding domain (LBD) of NMDAR1, respectively, and were synthesized by Biomatik (Figure 1A). The P2 peptide was emulsified with 
Complete Freund's Adjuvant (CFA) to immunize 2-month-old wildtype C57BL/6J male mice. To generate low titers of anti-NMDAR1 autoantibodies, mice received only a dose of primary immunization without a booster. Blood was collected via mouse tail vein at different time-points after the immunization (Figure 1B). Wildtype mouse paraffin brain sections were used for detection of anti-NMDAR1 autoantibodies by immunohistochemistry (IHC). Anti-NMDAR1 autoantibodies were detectable by IHC 3 weeks after immunization, and 5 out of 9 immunized mice developed the anti-NMDAR1 autoantibodies in blood by week 9 (WK9) (Figure 1C). To confirm the specificity of the anti-NMDAR1 autoantibodies, we conducted peptide blocking in IHC. As expected, binding of the anti-NMDAR1 autoantibodies to hippocampal NMDAR1 was blocked by the $\mathrm{P} 2$ peptide, but not by either the P1 peptide or bovine serum albumin (BSA) (Figure 1D). Optical intensities of CA1 staining (CA1 st oriens), after subtracting the background intensity from corpus callosum, were used as surrogates for the levels of the anti-NMDAR1 autoantibodies from WK3 to WK24. The levels of the anti-NMDAR1 autoantibodies varied a few folds between different mice at WK3 and were gradually stabilized by WK24 (Figure 1E). The titers of the anti-NMDAR1 autoantibodies in our mice (1 to 200 dilution in IHC) are 1-3 orders of magnitude lower than the titers of antiNMDAR1 autoantibodies in the blood of patients with anti-NMDAR1 encephalitis (1 to 2,000-500,000 dilution in IHC) (Gresa-Arribas et al., 2014). In this small male cohort, 5 males developed the anti-NMDAR1 autoantibodies, whereas 7 males did not have the anti-NMDAR1 autoantibodies (including 3 control males immunized with the CFA only).

To examine whether the anti-NMDAR1 autoantibodies against the P2 peptide can recognize NMDAR1 proteins in their native conformation, we conducted cell-based 
assays (Euroimmun) where NMDAR1 proteins were expressed on transfected HEK293 cells. Mouse serum containing the anti-NMDAR1 autoantibodies against the P2 peptide recognizes the NMDAR1 proteins on the transfected HEK293 cells (Figure 2A). As expected, immunohistochemical analysis using both human anti-NMDAR1 autoantibodies and mouse anti-NMDAR1 P2 autoantibodies demonstrated a complete co-localization of their staining (Figure 2B). After a series of dilutions of mouse serum, we found that titers of the anti-NMDAR1 autoantibodies against the P2 peptide range from 1:10-1:100 using cell-based assays among individual mice. These titers are comparable for low titers of blood anti-NMDAR1 autoantibodies in the general human population and psychiatric patients (Schou et al., 2016; Steiner et al., 2014), supporting the relevance of our mouse modeling for chronic effects of low titers of blood antiNMDAR1 autoantibodies on human mental health.

All of the mice carrying the anti-NMDAR1 autoantibodies were healthy. We examined several behaviors modulated by NMDAR in mice 24 weeks after immunization to investigate chronic effects of low titers of the anti-NMDAR1 autoantibodies. We found no differences in total distance traveled between the negative control mice and the mice carrying the anti-NMDAR1 autoantibodies against the P2 peptide (Figure 3A). No difference was observed in either center duration or center frequency (Figure 3B and 3C) between the two groups. We next examined sensorimotor gating as measured by prepulse inhibition between the two groups. There was no difference in either startle habituation (Figure 3D) or prepulse inhibition of startle (Figure 3E). Mouse cognitive function on spatial working memory and/or novelty detection was assessed with T-maze spontaneous alternation. A trend of impaired 
spontaneous alternation $(p=0.06)$ was found in mice carrying the anti-NMDAR1 autoantibodies (Figure 3F). Mice were further tested with fear conditioning to measure fear learning and memory as well as fear extinction. Mice carrying the anti-NMDAR1 autoantibodies appeared to acquire stronger fear memory by the end of acquisition in either post-shock (Figure 4A) or during the tone (CS) (Figure 4B). No difference was observed in contextual fear memory between the two groups (Figure 4C). However, mice carrying the anti-NMDAR1 autoantibodies had impaired cued fear extinction learning (Figure 4D) and recall of fear extinction (Figure 4E).

To enlarge the sample size and to include female mice, we purchased a large cohort of 40 2-month-old C57BL/6J mice (20 males, 20 females) for active immunization. To improve the success rate of production of the anti-NMDAR1 autoantibodies, we used Loss-of-Resistance (LOS) syringes (BD Epilor ${ }^{\mathrm{TM}}$ Syringe, 405291) to generate more viscous antigen/CFA emulsion. Half of mice (10 males, 10 females) were immunized with the P2 peptide emulsified with the CFA; whereas the other half (10 males, 10 females) were immunized with the CFA only to serve as the control group. A month later, anti-NMDAR1 autoantibodies were examined in mouse blood. All of the 20 mice immunized with the P2 peptide generated the anti-NMDAR1 autoantibodies (Figure 5), whereas none of the control mice produced the anti-NMDAR1 autoantibodies. Because of using the LOS syringes for emulsion, the success rate of the anti-NMDAR1 autoantibodies improved from $\sim 50 \%$ in the previous small cohort to $100 \%$ in this large cohort. All of the mice carrying the anti-NMDAR1 autoantibodies were healthy except for a control female mouse that died before behavioral analysis. Behavioral analysis was conducted 24 weeks after immunization to match the timeline of the previous small 
cohort (Figure 1B). A significant reduction of spontaneous alternation in T-maze was observed in mice carrying the anti-NMDAR1 autoantibodies (Figure 6A). After separating the sexes, we found that both the female and the male mice carrying the autoantibodies showed significant cognitive deficits in T-maze (Figure 6B), replicating the finding of the previous small male cohort. Statistical analyses of effect size $(d=1.29)$ and power (0.99) are summarized in Figure 6C. Mouse locomotor activities were examined using the Video-Tracker used in the small cohort. No difference was observed in total distance travelled (Figure 6D). There was a sex effect, but not the autoantibody effect, in center duration and center frequency (Figure 6E and 6F). No difference was found in either startle habituation (Figure 6G) or prepulse inhibition (Figure $6 \mathrm{H}$ ). Mice were further tested to confirm the fear conditioning phenotypes from the previous pilot male cohort. We did not observe differences in fear acquisition in either post-shock (Figure 7A) or during CS (Figure 7B). There was no autoantibody effect on contextual fear memory, except for a sex effect (Figure 7C). We detected a sex effect in fear extinction learning, but not an autoantibody effect (Figure 7D). No effect of the autoantibody was observed in recall of fear extinction (Figure 7E). The large cohort of mice replicated the T-maze deficit, but not the fear conditioning phenotypes, from the pilot cohort of male mice.

Since behavioral abnormalities are particularly susceptible for variations, we consider that another large cohort of mice is necessary for replication to conclude the effects of the anti-NMDAR1 autoantibodies on mouse behavioral phenotypes. We purchased the second large cohort of 40 2-month-old C57BL/6J mice (20 males, 20 females) for active immunization. The immunization was conducted exactly as 
described in the first large cohort. A month later, anti-NMDAR1 autoantibodies were examined in the blood of all 40 immunized mice. To reduce the use of research animals, we developed an in vitro One-Step quick assay for a rapid screening of the presence of anti-NMDAR1 autoantibodies (Figure 8A-8B). All of the 20 mice immunized with the P2 peptide generated the anti-NMDAR1 autoantibodies by the One-Step assay, whereas none of the 20 mice immunized with CFA only produced the anti-NMDAR1 autoantibodies. The One-Step assay showed 100\% consistence with immunohistochemical staining, providing an excellent alternative method for antibody screening without using mouse brain sections.

The same previous behavioral test battery was used for this large replication cohort of mice exception for two modifications. First, mouse locomotion was tested using Behavioral Pattern Monitor that is similar to the Video-Track but can additionally examine pokes and rears, the exploratory behaviors. Second, we shortened the interval between the immunization and the behavioral tests from 24 weeks to 11 weeks to delineate the timeline of the autoantibody effects on mouse cognitive functions. Defective spontaneous alternation in T-maze was validated again in mice carrying the anti-NMDAR1 autoantibodies with a $p$ value reaching 2.02E-08 (Figure 9A). Such a robust behavioral deficit can almost be used to differentiate which individual mice carrying the autoantibodies except for 5 mice overlapping with the control group. As expected, there is no sex effect, both female and male mice carrying the autoantibodies showed impaired spontaneous alternation in T-maze (Figure 9B). Statistical analyses of effect size ( $d=2.31)$ and power (1) are summarized in Figure 9C. Mouse locomotion and exploratory activities were investigated using Behavioral Pattern Monitor (BPM). 
There was no difference between the two groups of mice in total distance travelled (Figure 9D). There was no effect of the autoantibodies on center duration (9E), center frequency $(9 \mathrm{~F})$, pokes $(9 \mathrm{G})$, and rears $(9 \mathrm{H})$. No difference was found in either startle habituation (Figure 9I) or prepulse inhibition (Figure 9J). Mice were further tested with fear conditioning. We did not observe differences in fear acquisition in either post-shock (Figure 10A) or during CS (Figure 10B). There was no autoantibody effect on contextual fear memory (Figure 10C). We did not detect effect of the autoantibodies on fear extinction (Figure 10D). There was, however, a significant interaction $(F(12,420)=2.96, p=0.00056)$ between the autoantibodies and the CS in recall of fear extinction (Figure 10E).

Behavioral phenotypes from all 3 independent cohorts of mice are summarized and compared in Table I. Deficient spatial working memory and/or novelty detection in T-maze was consistently observed across all 3 cohorts of mice carrying the antiNMDAR1 autoantibodies. No differences in locomotion, exploration, startle, and prepusle inhibition were found between the control mice and mice carrying the autoantibodies. Fear conditioning phenotypes were not consistently detected in different cohorts of mice, suggesting that these phenotypes may be either mild or false positives by chance.

Anti-NMDAR1 autoantibodies against the P2 antigen persisted in mouse blood for more than 14 months. Since chronic presence of the circulating anti-NMDAR1 autoantibodies impairs mouse spatial working memory and/or novelty detection in Tmaze, we examined the brains of 5 pairs of mice for reduction of NMDAR1 proteins in different brain regions, particularly hippocampus. GFAP staining for astrogliosis and 
expression of GluR1 proteins were also examined in mouse hippocampus. We did not observe significant differences in the levels of NMDAR1, GluR1, and GFAP in hippocampus, cortex, and striatum between the two groups of mice (Figure 11).

\section{Discussion}

Our studies demonstrated that chronic presence of low titers of blood circulating anti-NMDAR1 autoantibodies against the P2 antigenic epitope is sufficient to specifically impair mouse spatial working memory and/or novelty detection in the integrity of bloodbrain barriers. To our knowledge, our studies are the first characterization of mice carrying anti-NMDAR1 autoantibodies against a single specific antigenic epitope. It is essential to investigate functions of anti-NMDAR1 autoantibodies at the level of individual antigenic epitopes, since anti-NMDAR1 autoantibodies against different antigenic epitopes may alter NMDAR functions differently. For example, antiNMDAR2A autoantibodies binding a specific antigenic epitope can function as an agonist (Chan et al., 2020). It will not be surprising if anti-NMDAR1 autoantibodies against certain antigenic epitopes may act as agonists rather than antagonists. Investigation of individual antigenic epitopes across the whole extracellular domain of NMDAR1 protein will help understand the effects of different anti-NMDAR1 autoantibodies on mouse behavioral phenotypes in future studies.

The mice carrying the anti-NMDAR1 autoantibodies against the P2 antigenic epitope were healthy and exhibited no abnormalities in a number of NMDAR-modulated behaviors except for robust deficient spatial working memory and/or novelty detection. Interestingly, intracerebroventricular injection of cerebrospinal fluid (CSF) from anti- 
NMDAR1 encephalitis patients causes robust impairment of mouse novelty detection without affecting locomotor activities and other behaviors (Planaguma et al., 2015). Effects of anti-NMDAR1 autoantibodies on mouse behaviors appear more restricted than either administration of NMDAR antagonists or NMDAR1 hypomorphic mice that display hyperlocomotion, deficient prepulse inhibition, and impaired context memory (Duncan et al., 2004; Geyer et al., 2001; Mohn et al., 1999). It will be interesting to know whether novelty detection and spatial working memory are more sensitive to NMDAR dysfunction than other NMDAR-modulated behaviors and cognition. Hippocampus plays a central role in novelty detection and spatial working memory. We examined expression of NMDAR1, GluR1, and GFAP in hippocampus using immunohistochemistry but did not find significant reduction of these proteins in mice carrying the anti-NMDAR1 autoantibodies. Our immunohistochemical analysis however may be not sensitive enough to identify subtle changes. It also cannot be ruled out that the blood circulating anti-NMDAR1 autoantibodies may preferentially infiltrate to localized brain regions to reduce NMDAR1 proteins that were not sampled by our brain sections.

Low titers of blood anti-NMDAR1 autoantibodies were found using cell-based assays in $\sim 10 \%$ of the general human population and psychiatric patients. By using the same cell-based assays, we found that the titers of blood anti-NMDAR1 autoantibodies against the P2 antigen in our mice are low and comparable to those in healthy humans. However, most anti-NMDAR1 autoantibodies are $\lg M$ and $\lg A$ isotypes in the blood of the general human population (Schou et al., 2016; Steiner et al., 2014), whereas antiNMDAR1 IgG and IgM autoantibodies are produced in our immunized mice. Therefore, 
it is important to investigate whether $\lg G$ or IgM anti-NMDAR1 autoantibodies are responsible for the deficient spatial working memory and/or novelty detection in mice immunized with the NMDAR1 P2 peptide antigens.

Behavioral phenotypes are particularly susceptible for non-replicability (Kafkafi et al., 2018). Differences in fear acquisition observed in the pilot cohort $(n 1=7, n 2=5)$ cannot be replicated by either of the two subsequent large cohorts of mice. Even between the two large cohorts of mice (each with 40 mice), sex effects on center duration, center frequency, and fear conditioning are not replicated with each other. Such non-replicability was also observed in recall of fear extinction between the two large cohorts. However, deficient spontaneous alternation in T-maze, a true behavioral phenotype by the autoantibodies, is replicated very well across all 3 different mouse cohorts, suggesting that more replications with large cohort sizes are necessary to remove potentially false positive behavioral phenotypes.

Anti-NMDAR1 autoantibodies have been found in patients with many diseases. $\sim 16 \%$ to $60 \%$ of patients with Alzheimer's Diseases or other dementias have antiNMDAR1 autoantibodies in their blood, but not in CSF (Doss et al., 2014). In addition to ovarian teratomas where anti-NMDAR1 encephalitis was originally identified, $20 \%$ of patients with a variety of other cancers develop anti-NMDAR1 autoantibodies in their blood, but not in CSF (Finke et al., 2017). In the general human population, prevalence of anti-NMDAR1 autoantibodies increases in blood during aging (Pan et al., 2018). Our studies suggest that chronic presence of blood circulating anti-NMDAR1 autoantibodies may be sufficient to cause cognitive impairments in both healthy persons and patients with a variety of diseases including psychiatric disorders. 
Acknowledgements: This work was supported by the grants R21MH123705 (XZ) and R21MH116186 (XZ) from the National Institute of Mental Health.

Author contributions: Conceived and designed the experiments: XZ. Performed the experiments: WY, SC, XZ. Analyzed the data: WY, SP, VR, XZ. Wrote the paper: XZ.

Conflict of interest: The authors declare no conflict of interest. 


\section{References}

Castillo-Gomez, E., Oliveira, B., Tapken, D., Bertrand, S., Klein-Schmidt, C., Pan, H., Zafeiriou, P., Steiner, J., Jurek, B., Trippe, R., et al. (2016). All naturally occurring autoantibodies against the NMDA receptor subunit NR1 have pathogenic potential irrespective of epitope and immunoglobulin class. Mol Psychiatry.

Chan, K., Nestor, J., Huerta, T.S., Certain, N., Moody, G., Kowal, C., Huerta, P.T., Volpe, B.T., Diamond, B., and Wollmuth, L.P. (2020). Lupus autoantibodies act as positive allosteric modulators at GluN2A-containing NMDA receptors and impair spatial memory. Nat Commun 11, 1403.

Dalmau, J., Gleichman, A.J., Hughes, E.G., Rossi, J.E., Peng, X., Lai, M., Dessain, S.K., Rosenfeld, M.R., Balice-Gordon, R., and Lynch, D.R. (2008). Anti-NMDA-receptor encephalitis: case series and analysis of the effects of antibodies. Lancet neurology 7 , 1091-1098.

Dalmau, J., Lancaster, E., Martinez-Hernandez, E., Rosenfeld, M.R., and Balice-Gordon, R. (2011). Clinical experience and laboratory investigations in patients with anti-NMDAR encephalitis. Lancet neurology 10, 63-74.

Doss, S., Wandinger, K.P., Hyman, B.T., Panzer, J.A., Synofzik, M., Dickerson, B., Mollenhauer, B., Scherzer, C.R., Ivinson, A.J., Finke, C., et al. (2014). High prevalence of NMDA receptor IgA/lgM antibodies in different dementia types. Ann Clin Transl Neurol 1, 822-832. 
Duncan, G.E., Moy, S.S., Perez, A., Eddy, D.M., Zinzow, W.M., Lieberman, J.A., Snouwaert, J.N., and Koller, B.H. (2004). Deficits in sensorimotor gating and tests of social behavior in a genetic model of reduced NMDA receptor function. Behav Brain Res 153, 507-519.

Finke, C., Bartels, F., Lutt, A., Pruss, H., and Harms, L. (2017). High prevalence of neuronal surface autoantibodies associated with cognitive deficits in cancer patients. $J$ Neurol 264, 1968-1977.

Fossan, G.O. (1977). The transfer of IgG from serum to CSF, evaluated by means of a naturally occurring antibody. Eur Neurol 15, 231-236.

Geyer, M.A., Krebs-Thomson, K., Braff, D.L., and Swerdlow, N.R. (2001).

Pharmacological studies of prepulse inhibition models of sensorimotor gating deficits in schizophrenia: a decade in review. Psychopharmacology (Berl) 156, 117-154.

Gresa-Arribas, N., Titulaer, M.J., Torrents, A., Aguilar, E., McCracken, L., Leypoldt, F., Gleichman, A.J., Balice-Gordon, R., Rosenfeld, M.R., Lynch, D., et al. (2014). Antibody titres at diagnosis and during follow-up of anti-NMDA receptor encephalitis: a retrospective study. Lancet neurology 13, 167-177.

Gresack, J.E., Risbrough, V.B., Scott, C.N., Coste, S., Stenzel-Poore, M., Geyer, M.A., and Powell, S.B. (2010). Isolation rearing-induced deficits in contextual fear learning do not require CRF(2) receptors. Behav Brain Res 209, 80-84. 
Halberstadt, A.L., van der Heijden, I., Ruderman, M.A., Risbrough, V.B., Gingrich, J.A., Geyer, M.A., and Powell, S.B. (2009). 5-HT(2A) and 5-HT(2C) receptors exert opposing effects on locomotor activity in mice. Neuropsychopharmacology 34, 1958-1967.

Hammer, C., Stepniak, B., Schneider, A., Papiol, S., Tantra, M., Begemann, M., Siren, A.L., Pardo, L.A., Sperling, S., Mohd Jofrry, S., et al. (2014). Neuropsychiatric disease relevance of circulating anti-NMDA receptor autoantibodies depends on blood-brain barrier integrity. Mol Psychiatry 19, 1143-1149.

Javitt, D.C., and Zukin, S.R. (1991). Recent advances in the phencyclidine model of schizophrenia. Am J Psychiatry 148, 1301-1308.

Ji, B., Wang, X., Pinto-Duarte, A., Kim, M., Caldwell, S., Young, J.W., Behrens, M.M., Sejnowski, T.J., Geyer, M.A., and Zhou, X. (2013). Prolonged Ketamine Effects in Hypomorphic Mice: Mimicking Phenotypes of Schizophrenia. PLoS ONE 8, e66327.

Jones, B.E., Tovar, K.R., Goehring, A., Jalali-Yazdi, F., Okada, N.J., Gouaux, E., and Westbrook, G.L. (2019). Autoimmune receptor encephalitis in mice induced by active immunization with conformationally stabilized holoreceptors. Sci Transl Med 11.

Kafkafi, N., Agassi, J., Chesler, E.J., Crabbe, J.C., Crusio, W.E., Eilam, D., Gerlai, R., Golani, I., Gomez-Marin, A., Heller, R., et al. (2018). Reproducibility and replicability of rodent phenotyping in preclinical studies. Neuroscience and biobehavioral reviews 87 , 218-232. 
Kim, M., Soontornniyomkij, V., Ji, B., and Zhou, X. (2012). System-wide immunohistochemical analysis of protein co-localization. PLoS ONE 7, e32043.

McCarthy, D.P., Richards, M.H., and Miller, S.D. (2012). Mouse models of multiple sclerosis: experimental autoimmune encephalomyelitis and Theiler's virus-induced demyelinating disease. Methods in molecular biology (Clifton, NJ 900, 381-401.

Mohn, A.R., Gainetdinov, R.R., Caron, M.G., and Koller, B.H. (1999). Mice with reduced NMDA receptor expression display behaviors related to schizophrenia. Cell 98, 427-436.

Pan, H., Oliveira, B., Saher, G., Dere, E., Tapken, D., Mitjans, M., Seidel, J., Wesolowski, J., Wakhloo, D., Klein-Schmidt, C., et al. (2018). Uncoupling the widespread occurrence of anti-NMDAR1 autoantibodies from neuropsychiatric disease in a novel autoimmune model. Mol Psychiatry.

Planaguma, J., Leypoldt, F., Mannara, F., Gutierrez-Cuesta, J., Martin-Garcia, E., Aguilar, E., Titulaer, M.J., Petit-Pedrol, M., Jain, A., Balice-Gordon, R., et al. (2015). Human N-methyl D-aspartate receptor antibodies alter memory and behaviour in mice. Brain 138, 94-109.

Risbrough, V., Ji, B., Hauger, R., and Zhou, X. (2014). Generation and Characterization of Humanized Mice Carrying COMT158 Met/Val Alleles. Neuropsychopharmacology.

Risbrough, V.B., Masten, V.L., Caldwell, S., Paulus, M.P., Low, M.J., and Geyer, M.A. (2006). Differential contributions of dopamine D1, D2, and D3 receptors to MDMA- 
induced effects on locomotor behavior patterns in mice. Neuropsychopharmacology 31, 2349-2358.

Schou, M., Saether, S.G., Borowski, K., Teegen, B., Kondziella, D., Stoecker, W., Vaaler, A., and Reitan, S.K. (2016). Prevalence of serum anti-neuronal autoantibodies in patients admitted to acute psychiatric care. Psychol Med 46, 3303-3313.

St-Amour, I., Pare, I., Alata, W., Coulombe, K., Ringuette-Goulet, C., Drouin-Ouellet, J., Vandal, M., Soulet, D., Bazin, R., and Calon, F. (2013). Brain bioavailability of human intravenous immunoglobulin and its transport through the murine blood-brain barrier. Journal of cerebral blood flow and metabolism : official journal of the International Society of Cerebral Blood Flow and Metabolism 33, 1983-1992.

Steiner, J., Teegen, B., Schiltz, K., Bernstein, H.G., Stoecker, W., and Bogerts, B. (2014). Prevalence of N-methyl-D-aspartate receptor autoantibodies in the peripheral blood: healthy control samples revisited. JAMA Psychiatry 71, 838-839.

Steiner, J., Walter, M., Glanz, W., Sarnyai, Z., Bernstein, H.G., Vielhaber, S., Kastner, A., Skalej, M., Jordan, W., Schiltz, K., et al. (2013). Increased prevalence of diverse Nmethyl-D-aspartate glutamate receptor antibodies in patients with an initial diagnosis of schizophrenia: specific relevance of IgG NR1a antibodies for distinction from N-methylD-aspartate glutamate receptor encephalitis. JAMA Psychiatry 70, 271-278.

Tarjinder Singh, B.M.N., Mark J. Daly (2020). Exome sequencing identifies rare coding variants in 10 genes which confer substantial risk for schizophrenia. MedRxiv. 
The Schizophrenia Working Group of the Psychiatric Genomics Consortium, S.R., James TR Walters, Michael C O'Donovan (2020). Mapping genomic loci prioritises genes and implicates synaptic biology in schizophrenia. MedRxiv.

Toth, M., Gresack, J.E., Bangasser, D.A., Plona, Z., Valentino, R.J., Flandreau, E.I., Mansuy, I.M., Merlo-Pich, E., Geyer, M.A., and Risbrough, V.B. (2014). Forebrainspecific CRF overproduction during development is sufficient to induce enduring anxiety and startle abnormalities in adult mice. Neuropsychopharmacology 39, 1409-1419.

Wang, Q., Delva, L., Weinreb, P.H., Pepinsky, R.B., Graham, D., Veizaj, E., Cheung, A.E., Chen, W., Nestorov, I., Rohde, E., et al. (2018). Monoclonal antibody exposure in rat and cynomolgus monkey cerebrospinal fluid following systemic administration. Fluids Barriers CNS 15, 10.

Zhou, X. (2021). A novel one-step quick assay for detection of SARS-CoV2 antibodies across mammalian species. PeerJ 9, e11381. 


\section{Figure Legend}

Figure 1. Generation of anti-NMDAR1 autoantibodies in mice. (A) P1 and P2 peptide antigens, each 20 amino acids long, were selected and synthesized from amino terminal domain (ATD) and ligand binding domain (LBD) of NMDAR1, respectively. TMD: transmembrane domain. CTD: C-terminal domain. (B) After immunization, blood was collected at different timepoints from week 3 (WK3) to week 24 (WK24). Behavioral studies were conducted after the last blood collection. (C) Wildtype mouse paraffin brain sections were used for detection of anti-NMDAR1 autoantibodies against the P2 antigen. Blood of immunized mice was diluted at 1:200 for the IHC analysis. CTRL: hippocampal staining with a commercial mouse anti-NMDAR1 monoclonal antibody (1:40,000 dilution) as the positive control. At week 4 (WK4), \#3, \#6, \#7, and \#9 mice were positives for anti-NMDAR1 autoantibodies. At week 9 (WK9), \#3, \#4, \#6, \#7, and \#9 were positives. (D) Pre-incubation of the P2 antigen with positive mouse sera blocked the staining of mouse hippocampus, whereas pre-incubation of either the P1 antigen or BSA had no blocking effects on the staining. (E) Differences of optical intensities between hippocampal CA1 st oriens and corpus callosum were quantified with Image $\mathrm{J}$ and served as surrogates for the levels of anti-NMDAR1 autoantibodies in different mice from week 3 to week 24 .

\section{Figure 2. Characterization of the anti-NMDAR1 autoantibodies using cell-based}

assays. (A) Human NMDAR1 proteins were expressed on HEK293 cells on BIOCHIPs purchased from Euroimmun. Both anti-Human NMDAR1 autoantibody (Euroimmun) and mouse anti-NMDAR1 antibody (BD, diluted at 1:10,000) recognize the NMDAR1 
proteins on HEK293 cells in cell-based assays. The anti-NMDAR1 autoantibodies

(diluted at 1:10) against the P2 peptide antigens from our mice also recognize the native conformation of the NMDAR1 proteins in cell-based assays. (B) Co-localization of the staining between the anti-Human NMDAR1 and mouse anti-NMDAR1 P2 autoantibodies in cell-based assays.

Figure 3. Behavioral analysis of the mice in open field locomotion, prepulse inhibition, and T-maze tests. Negative control male mice $(n=7)$; positive NMDAR1 male mice $(n=5)$. (A) No difference $(F(1,10)=0.45, p=0.52)$ in total distances traveled was observed between the two groups, 10 min for each time-block. (B) There is no difference $(F(1,10)=0.06, p=0.81)$ in center duration between the two groups. (C) No difference $(F(1,10)=0.38, p=0.55)$ was observed between the two groups in frequencies for mice entering the center. (D) There is no difference $(F(1,10)=0.07, p=0.8)$ in startle habituation between the two groups of mice. $(E)$ No difference $(F(1,10)=0.05, p=0.84)$ was observed in prepulse inhibition across pp4, pp8, pp16 prepulse levels between the two groups. (F) Alternation percentages of individual mice were present as boxplot. There was a trend $(\mathrm{t}(10)=1.81, \mathrm{p}=0.06$, one-tail $t$ test) of lower spontaneous alternation in T-maze in mice carrying the anti-NMDAR1 autoantibodies. All data are present as Mean+SEM.

Figure 4. Fear extinction. 4-day fear conditioning tests were conducted as previously described (Risbrough et al., 2014). On Day 1 fear acquisition, mice carrying the antiNMDAR1 autoantibodies displayed higher freezing percentages after shock (A, 
$F(1,10)=7.06, p=0.024)$ or during the tone $(B, F(1,10)=7.03, p=0.024)$. (C) On Day 2, contextual fear memory was assessed. Freezing percentages were calculated for the first two blocks, 2 min for each block. There was no difference $(F(1,10)=0.002, p=0.97)$ between the two groups. (D) On Day 3, fear extinction was assessed with 32 tones (CS) without foot shock. Slower fear extinction $(F 91,10)=19.35, p=0.001)$ was observed in mice carrying the anti-NMDAR1 autoantibodies. CS0: pre-tone. (E) On Day 4, recall of fear extinction was examined with $12 \mathrm{CS}$ without foot shock. An impaired recall of fear extinction $(F(1,10)=7.9, p=0.019)$ was detected in mice carrying the anti-NMDAR1 autoantibodies. CS0: pre-tone. All data are present as Mean+SEM.

Figure 5. Generation of the anti-NMDAR1 autoantibodies in a large cohort. A large cohort of 40 mice (20 males and 20 females) was immunized with either the P2 plus the CFA or the CFA only. After improving the antigen emulsification using the LOS syringes, all mice (10 males and 10 females) immunized with the P2 plus the CFA generated antiNMDAR1 autoantibodies. No difference was observed between the males and females. All of the control mice (10 males and 10 females) immunized with the CFA only are negative for the autoantibodies against the NMDAR1 P2 antigen.

Figure 6. Impaired spontaneous alternation in T-maze. In the large cohort of 40 immunized mice, one female control mice immunized with the CFA only died before behavioral analysis. All other mice are healthy. (A) Alternation percentages of individual mice were present as boxplots. A significant reduction of spontaneous alternation was replicated in the large cohort $(F(1,35)=15.96, p=0.0003)$. (B) Both male 
and female mice carrying the anti-NMDAR1 autoantibodies displayed impaired spontaneous alternation, replicating the T-maze performance of the previous small male cohort. (C) This large cohort of mice provided a sufficient statistical power (0.99) to analyze the effect size $(d=1.296)$ of the anti-NMDAR1 autoantibodies between the mice with or without the anti-NMDAR1 autoantibodies. (D) There is neither $\operatorname{sex}(F(1,31)=2.18$, $p=0.15)$ nor autoantibody $(F(1,31)=0.7, p=0.41)$ effect on total distance travelled in the open field. (E) Male mice spent significant more time $(F(1,31)=10.37, p=0.003)$ in the center than female mice, but there is no autoantibody effect $(F(1,31)=1.05, p=0.31)$. Consistent with the center time, a significant more center frequency $(F(1,31)=6.38$, $p=0.017)$ was observed in male mice. There is no effect of the autoantibody $(F(1,31)=0.99, p=0.33) .(\mathbf{G})$ There is neither $\operatorname{sex}(F(1,35)=1.11, p=0.3)$ nor autoantibody $(F(1,35)=0.01, p=0.93)$ effect in startle habituation. No interactions between startle blocks and autoantibody $(F(3,105)=1.57, p=0.2)$. (H) Neither $\operatorname{sex}(F(1,35)=0.3, p=0.57)$ nor autoantibody $(F(1,35)=0.93, p=0.34)$ effect was observed in PPI. All data are present as Mean+SEM.

Figure 7. No effects of the anti-NMDAR1 autoantibodies on fear conditioning. (A) In Day1 fear acquisition, there is neither $\operatorname{sex}(F(1,31)=0.35, p=0.56)$ nor autoantibody $(F(1,31)=0.51, p=0.48)$ effect on post-shock freezing time. (B) Neither sex $(F(1,31)=1.93, p=0.17)$ nor autoantibody $(F(1,31)=0.61, p=0.44)$ effect was observed during CS tone. (C) In Day2 context memory, female mice have significant higher freezing percentages $(F(1,35)=5.2, p=0.029)$ than the male mice, but there is no autoantibody effect $(F(1,35)=0.9, p=0.35)$. (D) In Day3 fear extinction, there is a trend 
of sex effect $(F(1,35)=2.94, p=0.095)$, but without autoantibody effect $(F(1,35)=0.04$, $p=0.85)$. CS0: pre-tone. (E) In Day4 recall of fear extinction, neither sex $(F(1,35)=0.24$, $p=0.63)$ nor autoantibody $(F(1,35)=0.24, p=0.63)$ effect was observed. CS0: pre-tone. All data are present as Mean+SEM.

\section{Figure 8. Generation of the anti-NMDAR1 autoantibodies in a large replication}

cohort. Another large cohort of 40 mice (20 males and 20 females) was immunized with either the P2 plus the CFA or the CFA only. (A) A new One-Step quick assay was developed to screening the presence of the anti-NMDAR1 P2 autoantibodies in the blood of all 40 immunized mice. Either the P2 peptide or the LBD of NMDAR1 was fused with GFP. The fusion proteins were purified from E coli for the assay. The protein A/G/L instantly aggregates antibody-antigen-GFP complexes that emit strong green fluorescence (Zhou, 2021). (B) All of the 20 mice immunized with the P2 peptide antigens developed anti-NMDAR1 autoantibodies one month after immunization, whereas all of the 20 mice immunized with CFA only were negative.

Figure 9. Replication of deficient spontaneous alternation in T-maze. All mice are healthy. (A) Alternation percentages of individual mice were present as boxplots. Spontaneous alternation was dramatically impaired in mice carrying the anti-NMDAR1 autoantibodies $(F(1,36)=51.3, p=2.02 E-08)$, replicating our finding across all 3 different mouse cohorts. (B) Both male and female mice carrying the anti-NMDAR1 autoantibodies displayed impaired spontaneous alternation, replicating the T-maze performance of the previous cohorts. (C) This large replication cohort of mice provided a sufficient statistical power (1) to analyze the effect size $(d=2.31)$ of the anti-NMDAR1 
autoantibodies between the mice with or without the anti-NMDAR1 autoantibodies.

Mouse locomotion and exploratory activity were examined using Behavioral Pattern Monitor. One control mice died during the behavioral tests. (D) There is neither sex $(F(1,35)=0.092, p=0.76)$ nor autoantibody $(F(1,35)=0.44, p=0.51)$ effect on total distance travelled in the open field. (E) Neither sex effect $(F(1,35)=0.054, p=0.82)$ nor the autoantibody effect $(F(1,35)=1.33, p=0.26)$ was found for center duration between the group of mice. $(F)$ There is neither $\operatorname{sex}(F(1,35)=0.021, p=0.88)$ nor autoantibody $(F(1,35)=0.58, p=0.45)$ effect on center frequency. $(\mathbf{G})$ Neither sex $(F(1,35)=0.05$, $p=0.83)$ nor autoantibody $(F(1,35)=0.23, p=0.63)$ effect on pokes in Behavioral Pattern Monitor test. $(\mathrm{H})$ There is a sex effect $(F(1,35)=5.4, p=0.025)$, but no autoantibody effect $(F(1,35)=0.04, p=0.84)$ on rears. (I) There is no $\operatorname{sex}(F(1,35)=0.17, p=0.68)$ or autoantibody $(F(1,35)=0.09, p=0.77)$ effect on startle habituation. No interactions between startle blocks and autoantibody $(F(3,105)=1.1, p=0.35)$ was observed. (J) Neither $\operatorname{sex}(F(1,35)=1.45, p=0.24)$ nor autoantibody $(F(1,35)=0.03, p=0.87)$ effect was observed in PPI. All data are present as Mean+SEM.

Figure 10. Effects of the anti-NMDAR1 autoantibodies on fear conditioning in the large replication cohort. (A) In Day 1 fear acquisition, there is neither sex $(F(1,36)=0.16, p=0.69)$ nor autoantibody $(F(1,36)=0.45, p=0.51)$ effect on post-shock freezing time. (B) Neither $\operatorname{sex}(F(1,36)=1.79, p=0.19)$ nor autoantibody $(F(1,36)=0.53$, $p=0.47$ ) effect was observed during CS tone. (C) In Day 2 context memory, neither sex $(F(1,35)=2.05, p=0.16)$ nor autoantibody $(F(1,35)=0.55, p=0.46)$ effect was observed. (D) In Day 3 fear extinction, there is no sex effect $(F(1,35)=0.25, p=0.62)$ or autoantibody effect $(F(1,35)=0.52, p=0.48)$. CS0: pre-tone. $(E)$ In Day 4 recall of fear 
extinction, neither $\operatorname{sex}(F(1,35)=1.26, p=0.27)$ nor autoantibody $(F(1,35)=2, p=0.17)$

effect was observed. However, there is a significant interaction $(F(12,420)=2.96$, $p=0.00056$ ) between the autoantibody and CS. CS0: pre-tone. All data are present as Mean+SEM.

Figure 11. Lack of significant differences of NMDAR1, GluR1, and GFAP in the brains of mice chronically carrying the anti-NMDAR1 autoantibodies. 5 pairs of mice with or without the anti-NMDAR1 autoantibodies were sacrificed to examine expression of NMDAR1, GluR1, and GFAP in hippocampus, cortex, and striatum. Representative staining of two mice from each group are shown. Image $\mathrm{J}$ was used to quantify the optical densities of the staining at different brain regions. There are no significant differences in expression of NMDAR1, GluR1, and GFAP between the control mice and the mice carrying the anti-NMDAR1 autoantibodies. Data were presented as mean+SEM. 


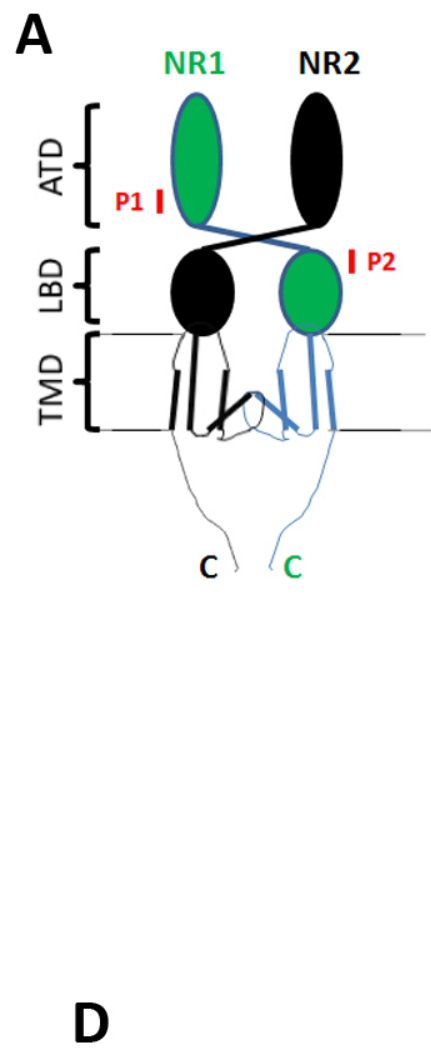

Hippocampus

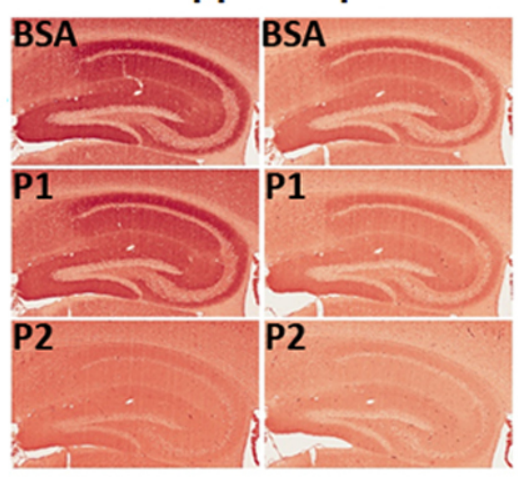

C
B Immunization

Behavioral Analysis
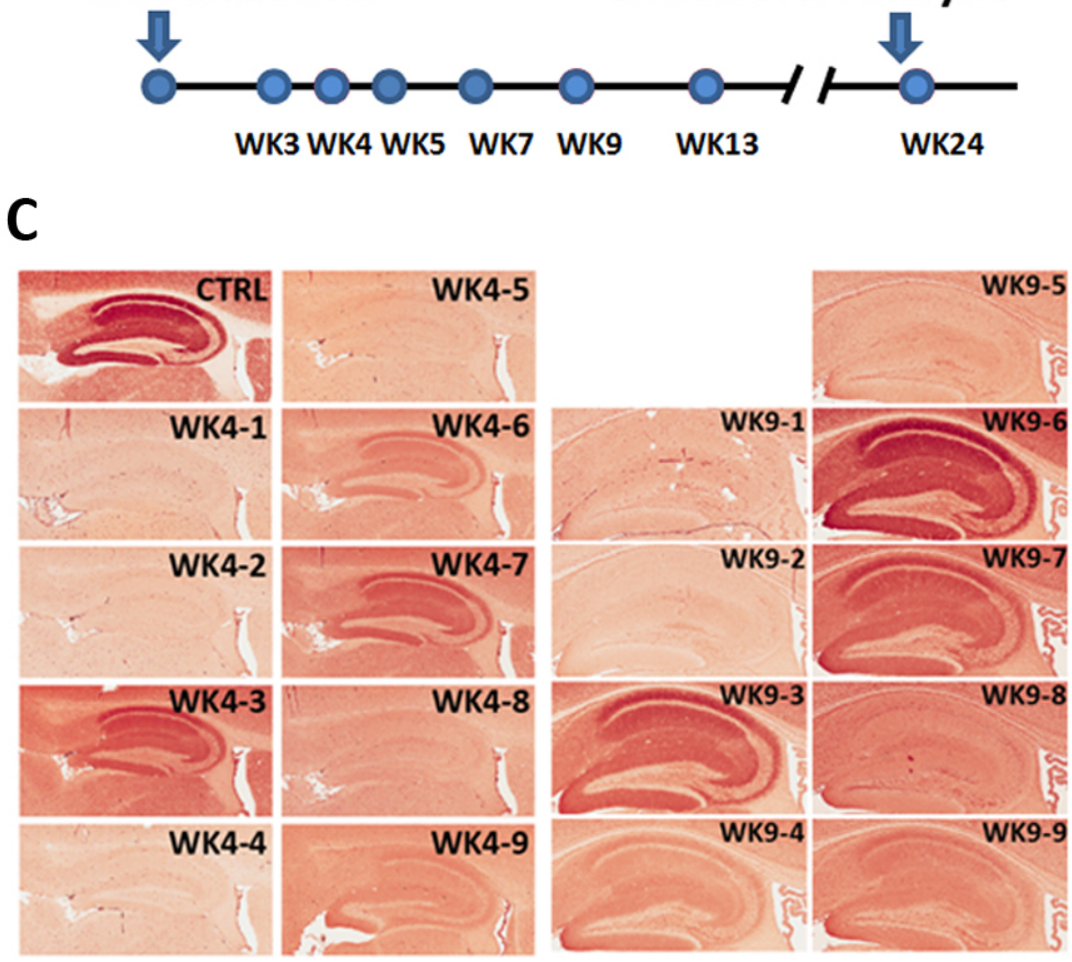

Dynamics of Autoantibodies

E

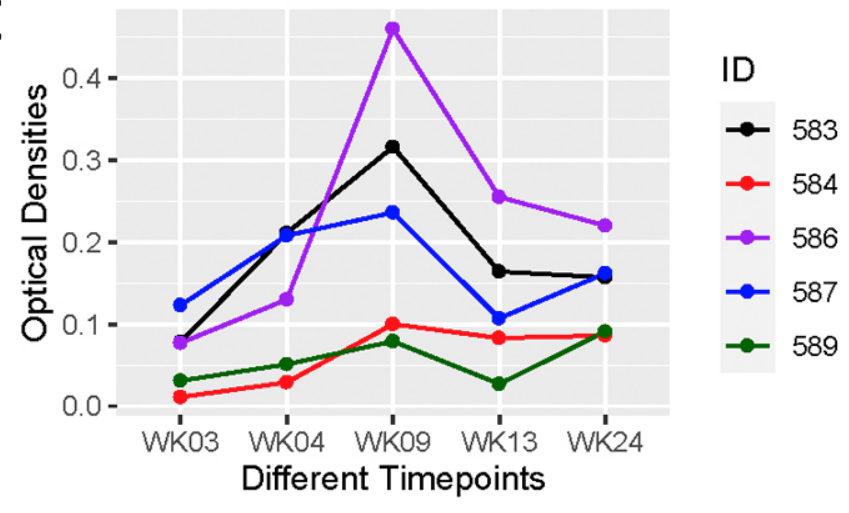

Figure 1 


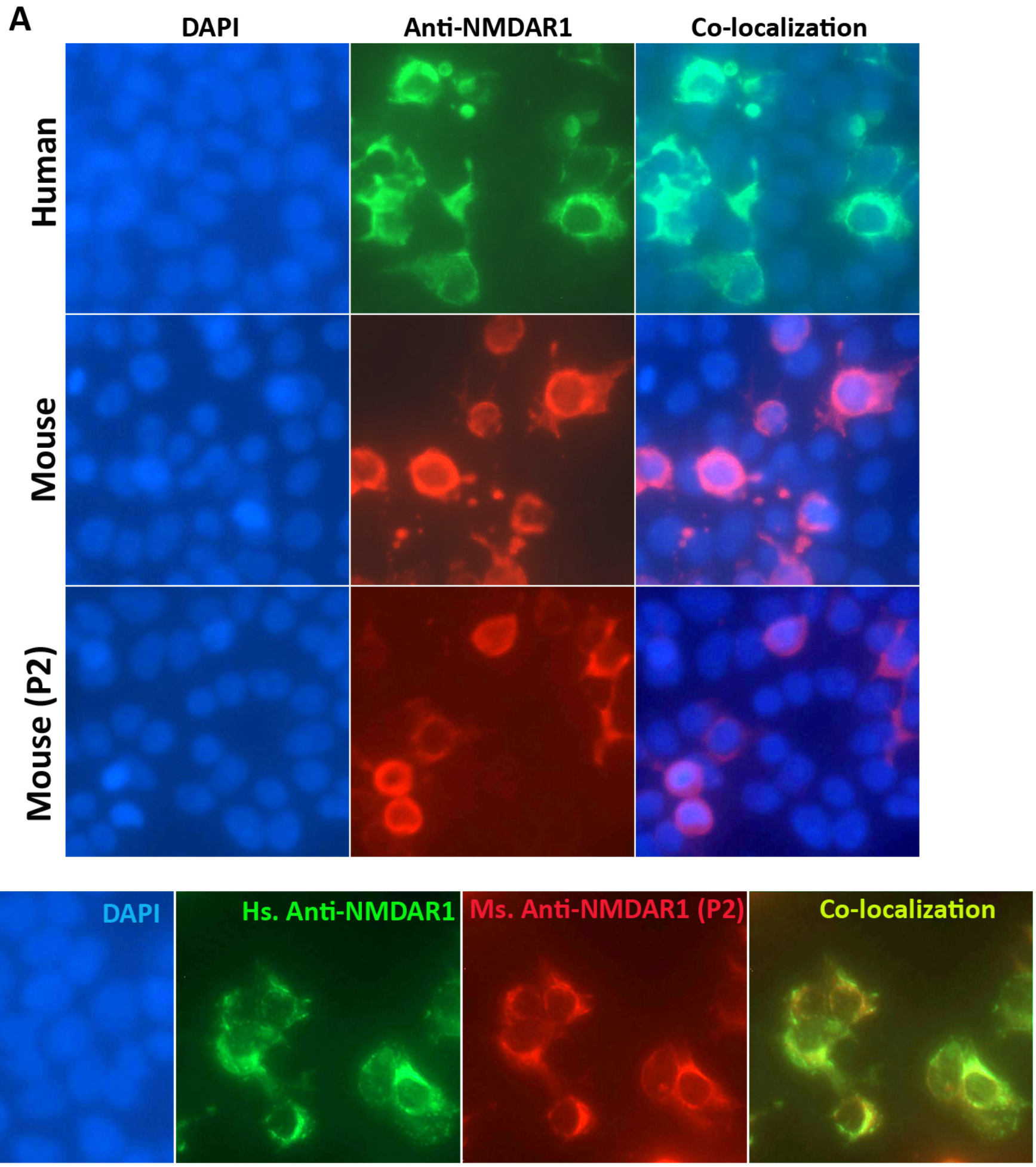

Figure 2 
A

Distance Traveled

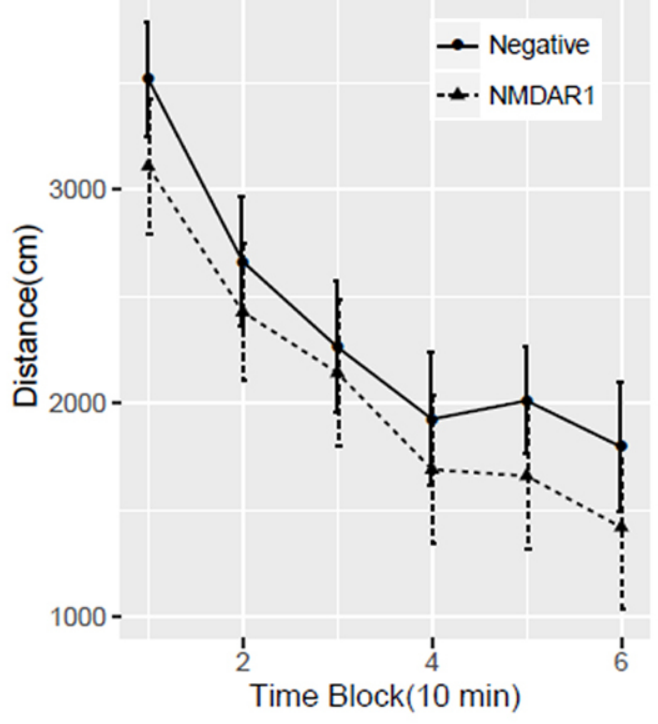

B

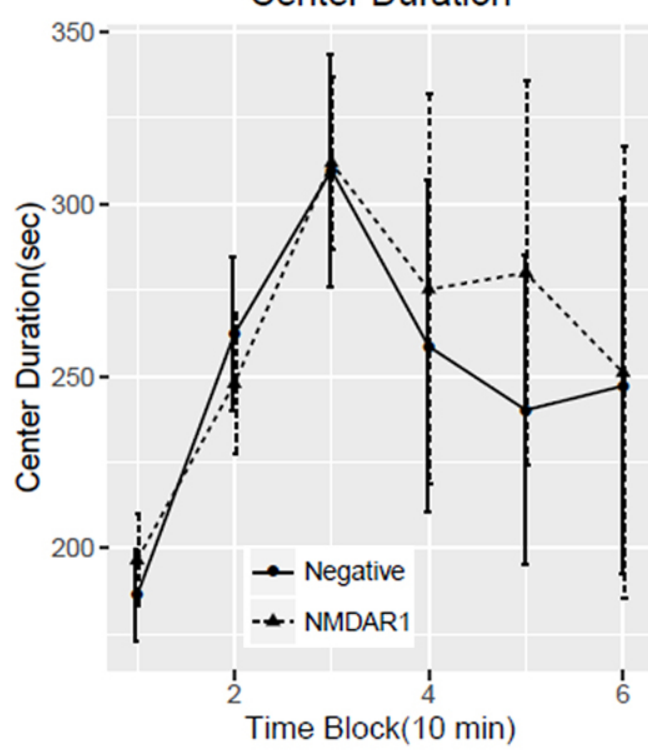

C

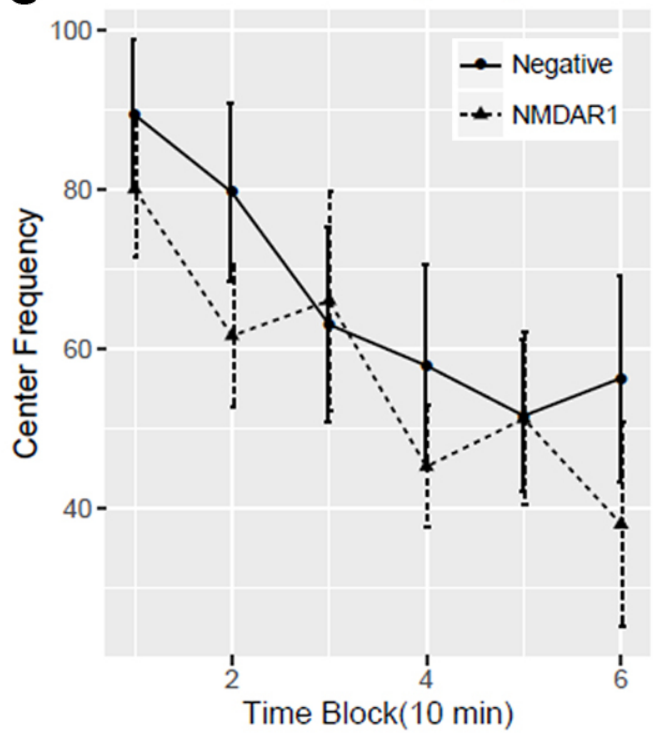

D

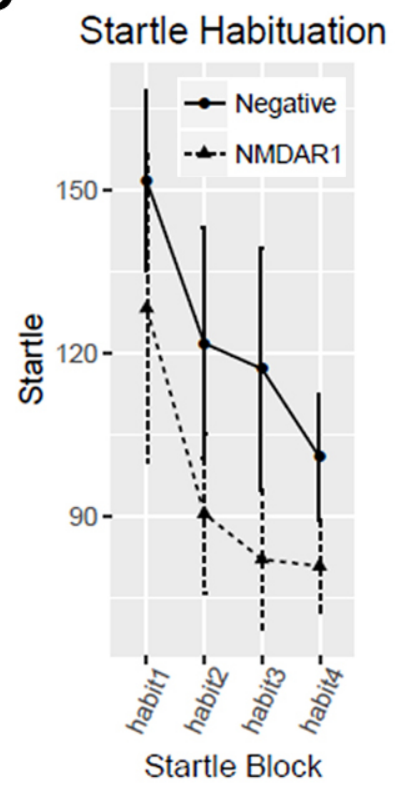

E Prepulse Inhibition

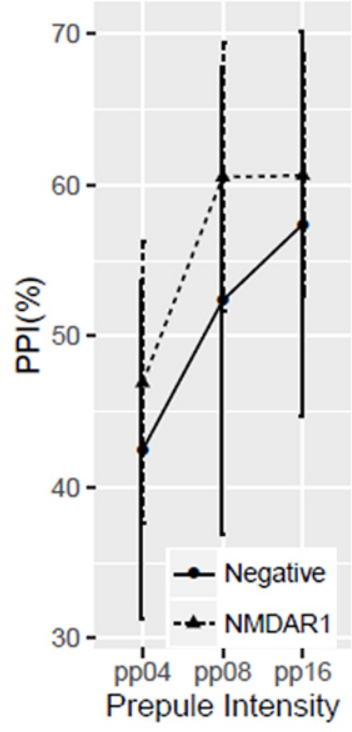

F T-Maze

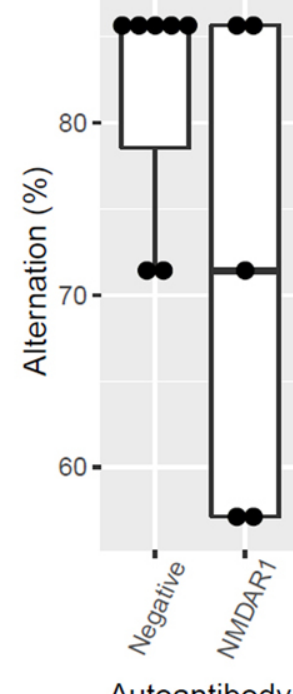

Autoantibody

Figure 3 
A Acquisition_Postshock

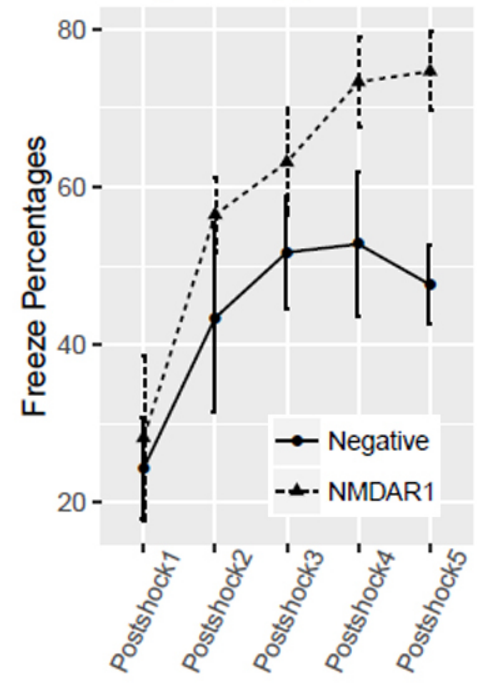

D

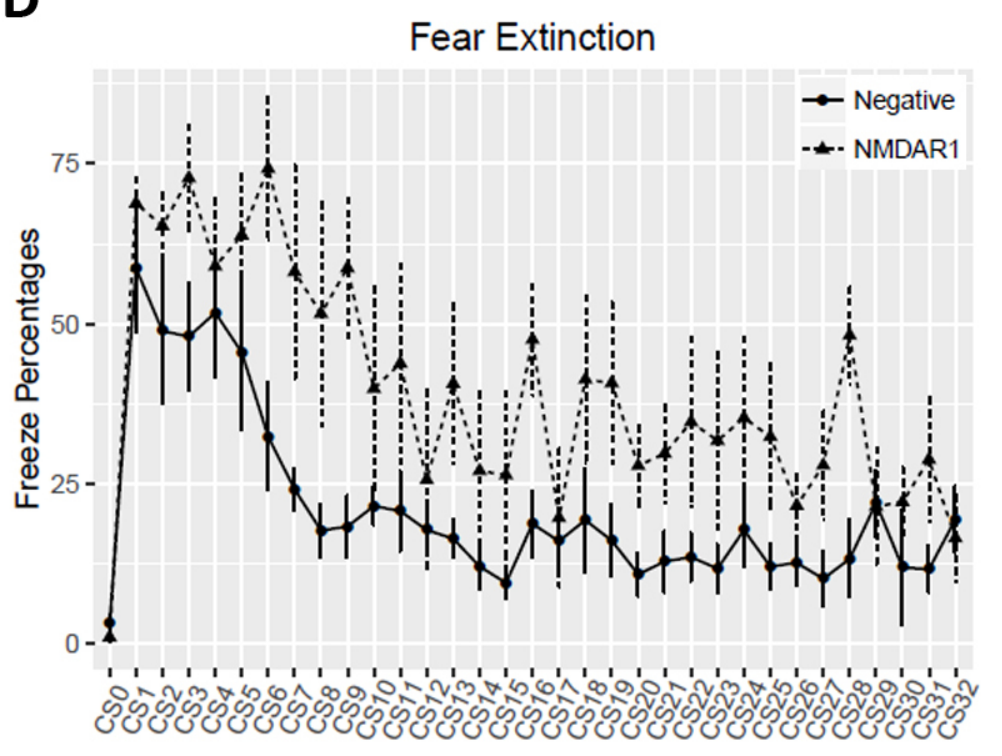

B Acquisition_CS

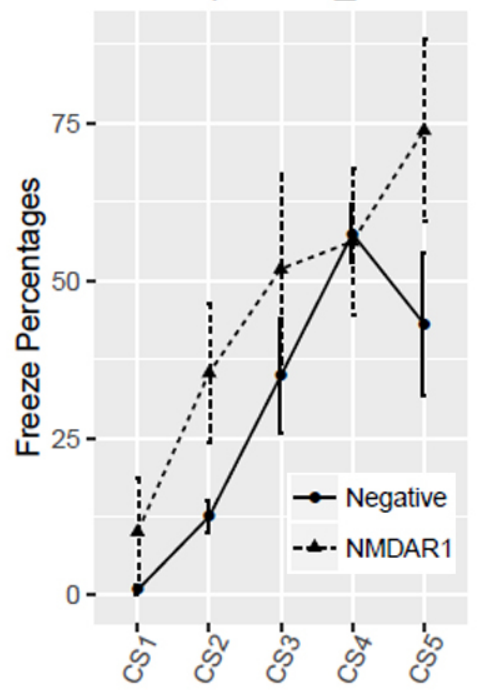

C Context Memory

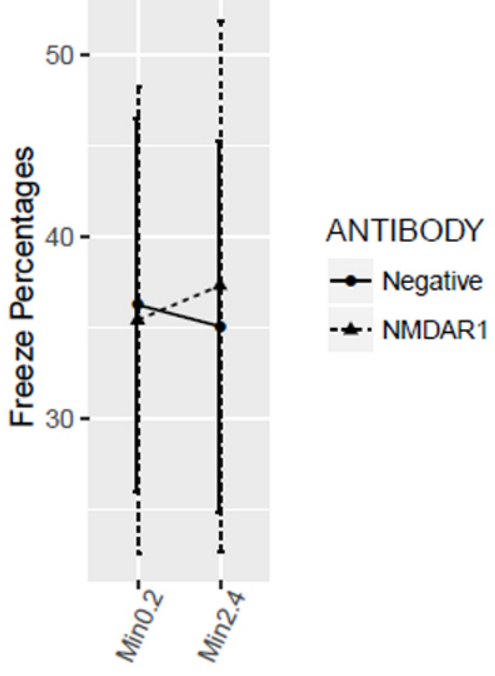

E

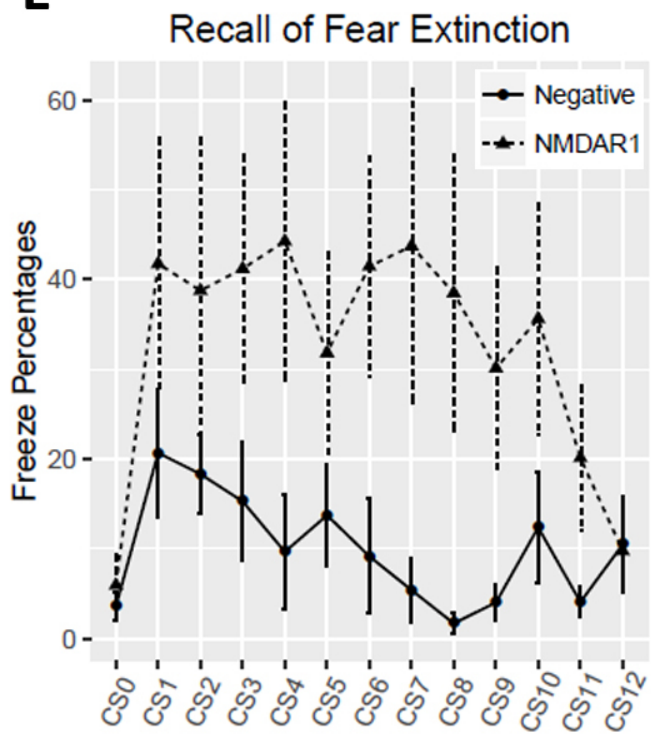

Figure 4 


\section{Positive}

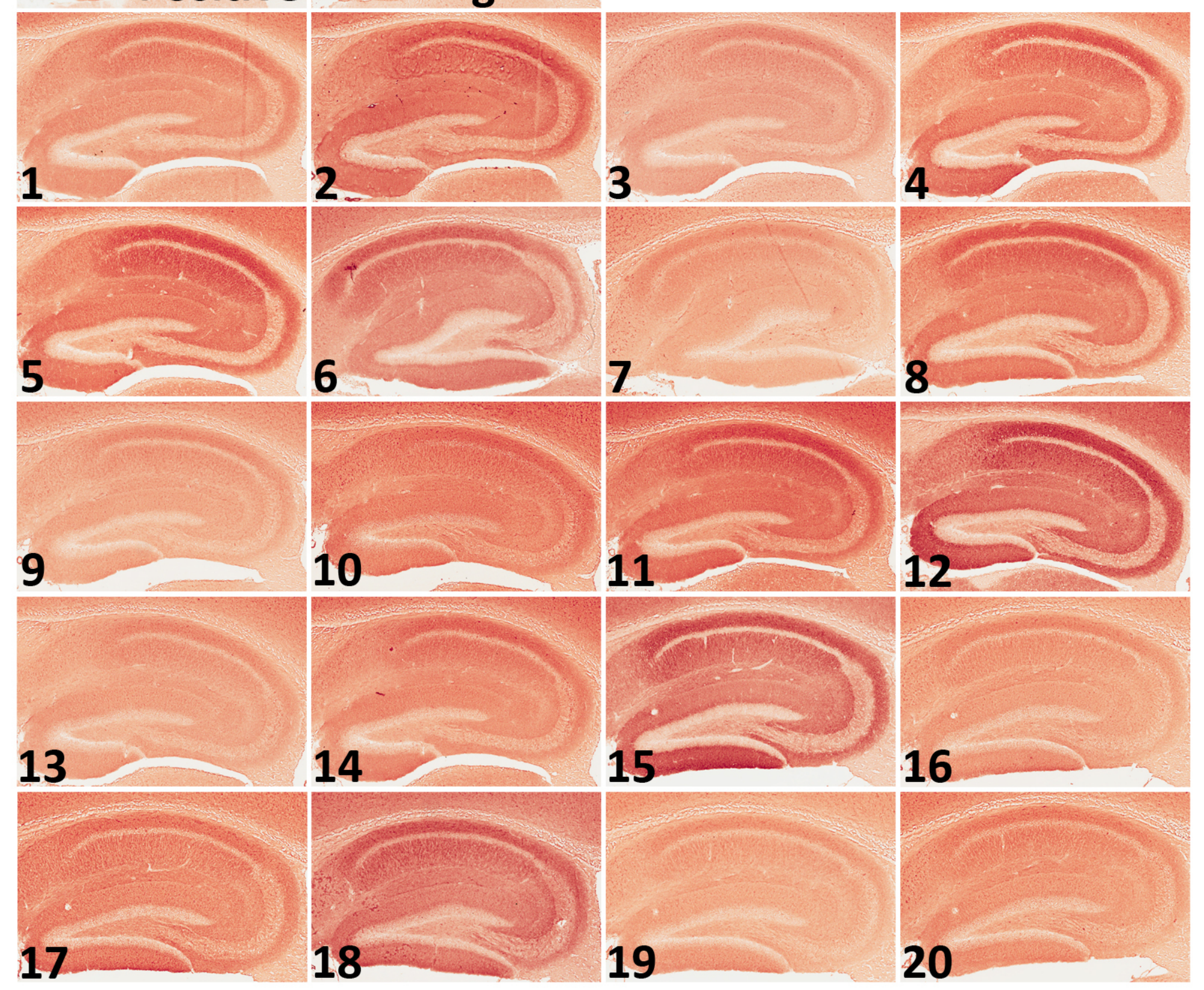

Figure 5 


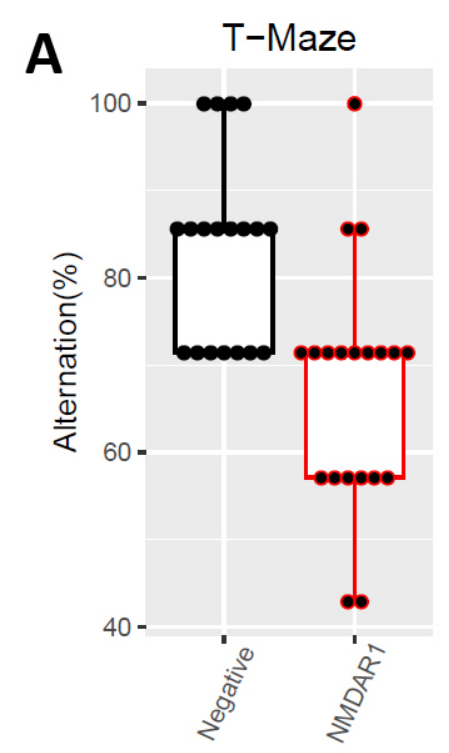

Autoantibody

D Distance Traveled

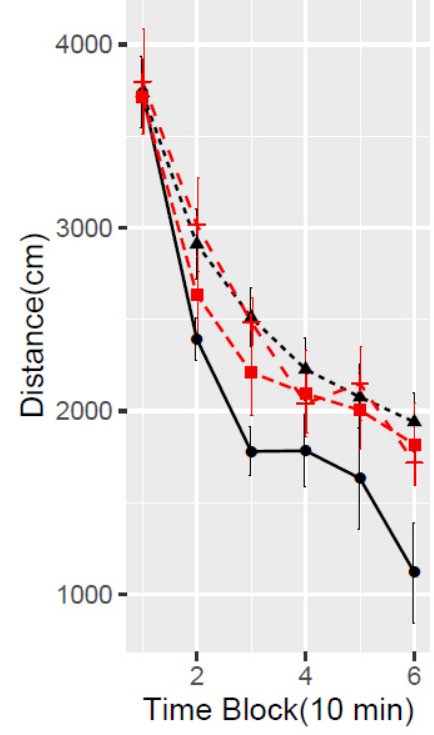

G

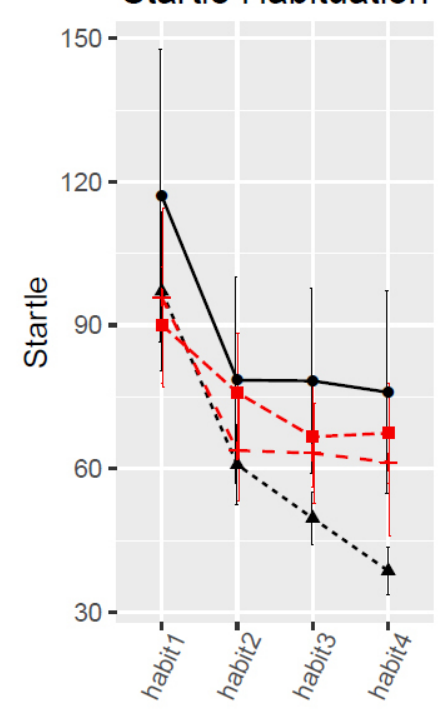

Startle Block

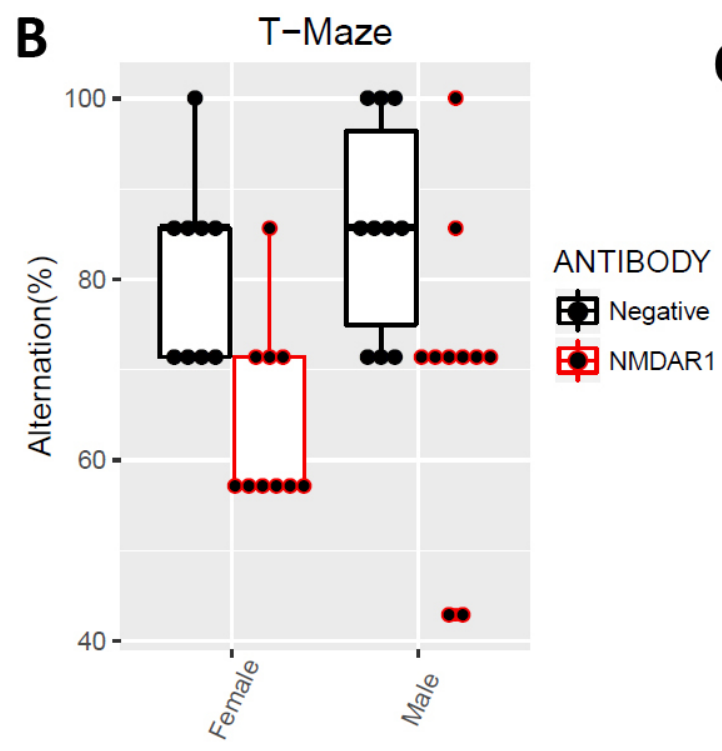

SEX

E

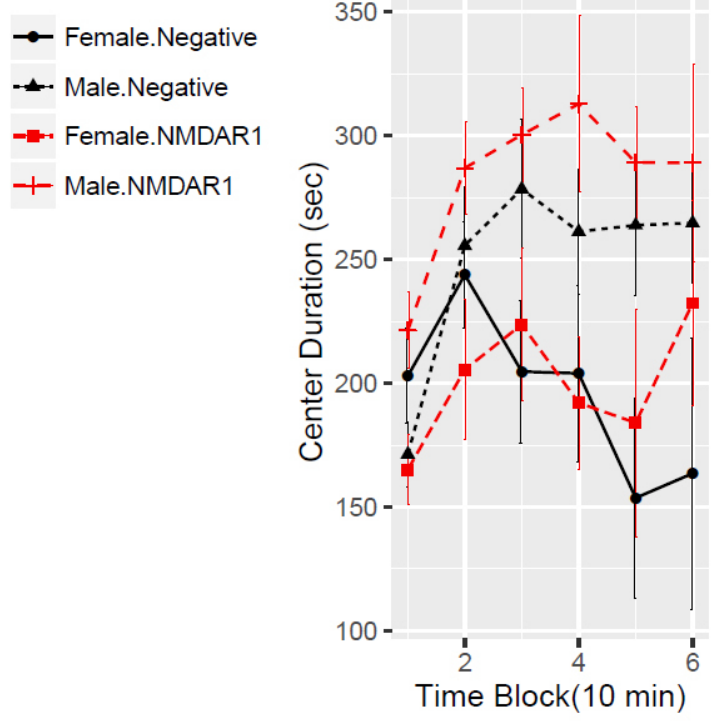

H Prepulse Inhibition

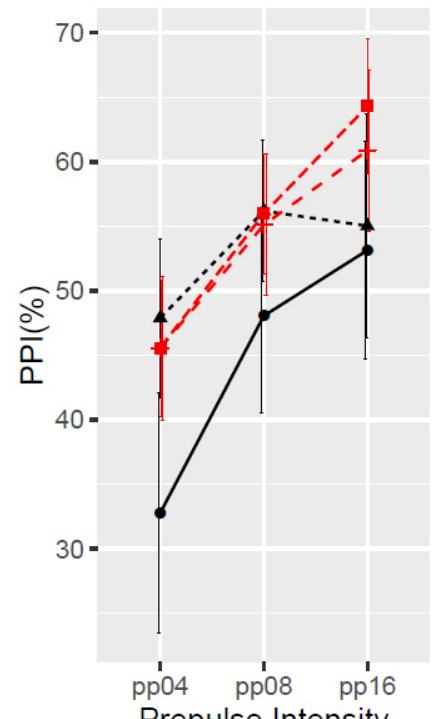

Prepulse Intensity
T-Maze Power Analsysis

\begin{tabular}{l|r}
\hline Sample size & $\begin{array}{l}\text { Negative }=19 \\
\text { NMDAR1 }=20\end{array}$ \\
\hline ANOVA F & $F(1,35)=15.961$ \\
$p$ value & 0.0003 \\
Effect size (d) & 1.296 \\
\hline Power & 0.99 \\
\hline
\end{tabular}

F Center Frequency

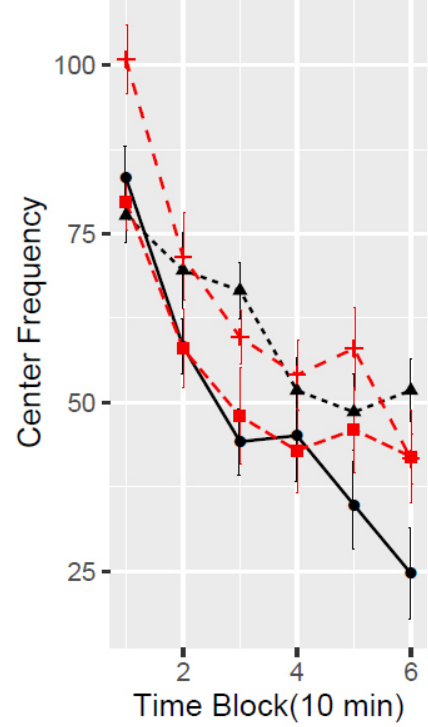


A Acquisition(Postshock)

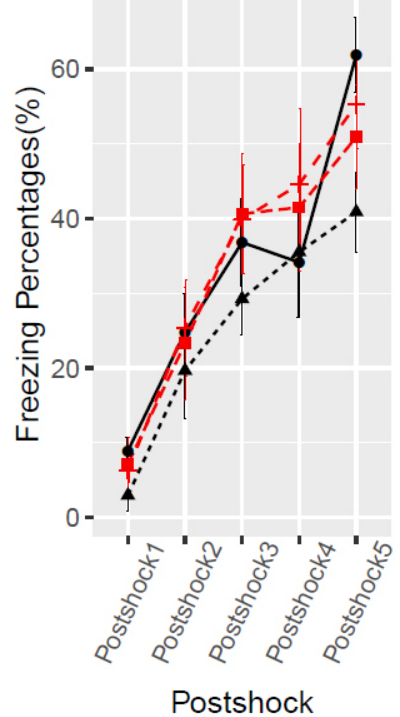

D

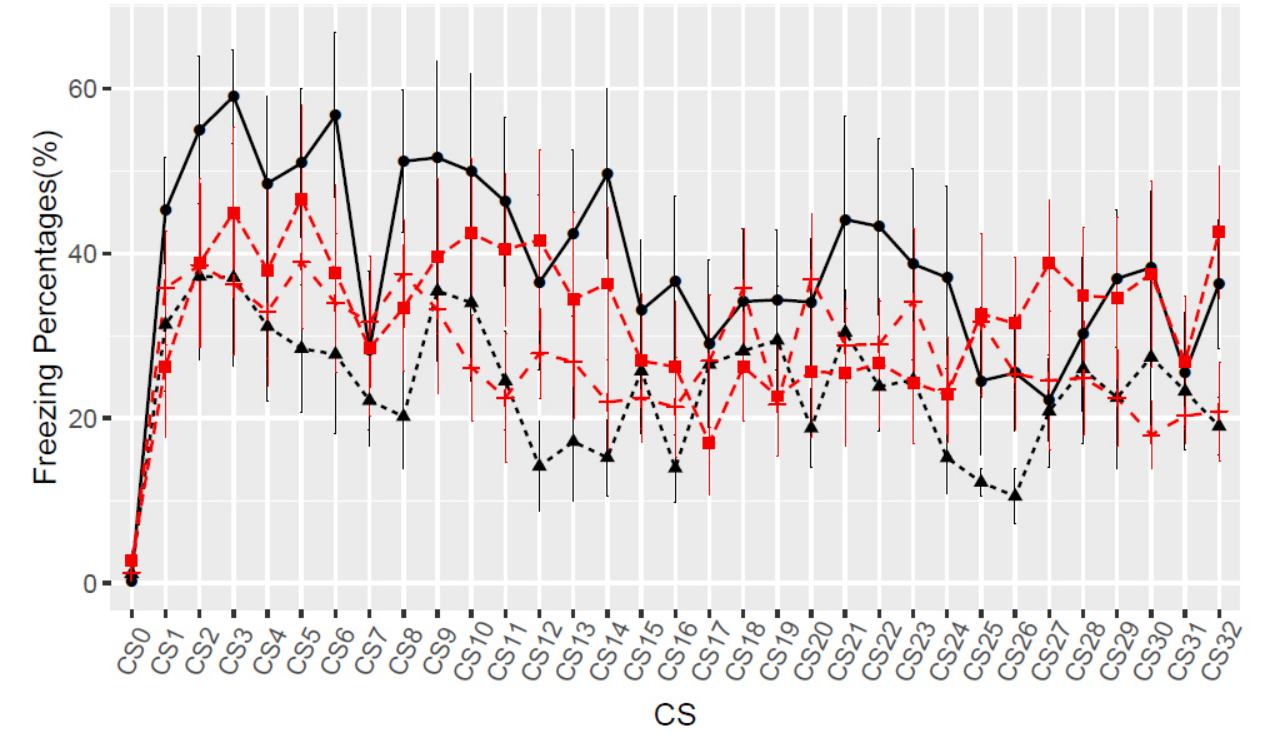

C Context Memory

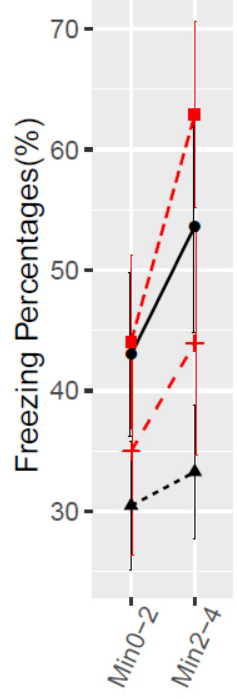

E Recall of Fear Extinction

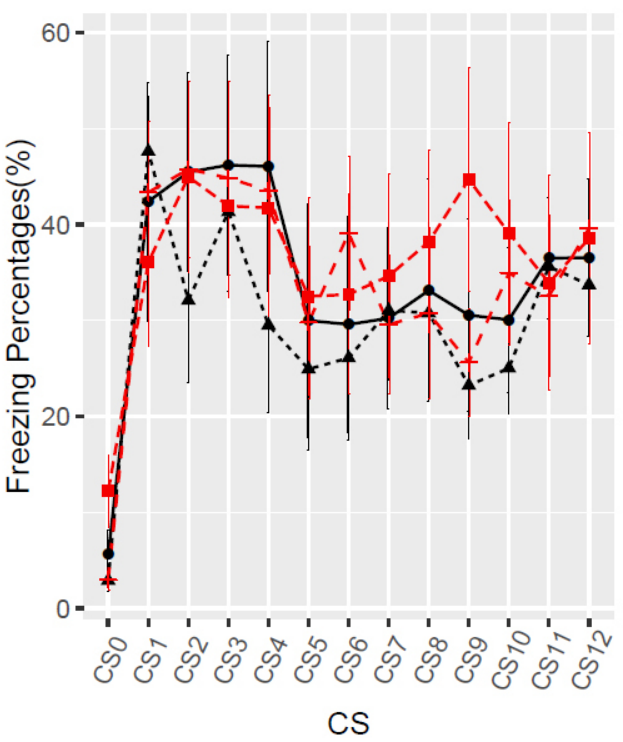


A

B
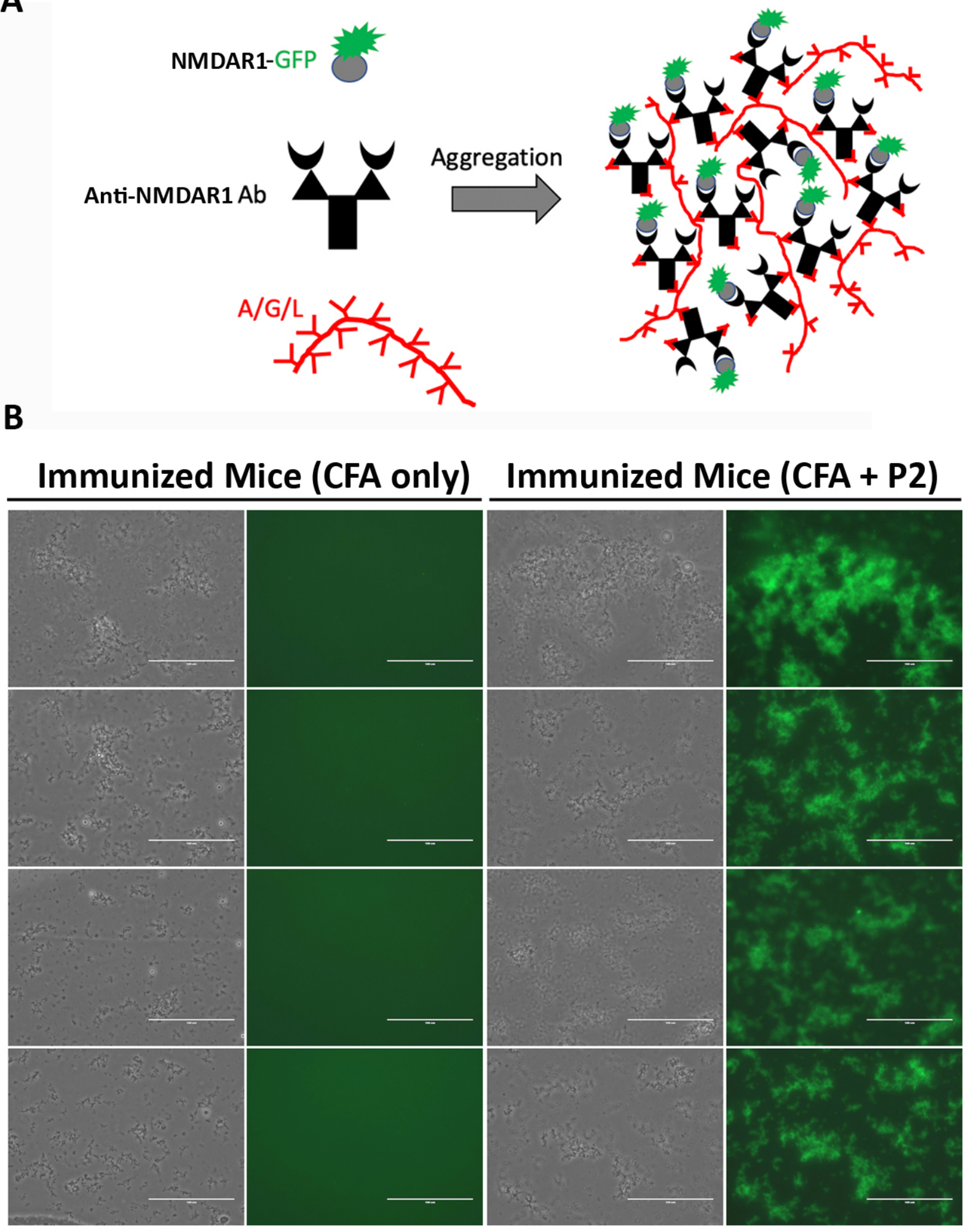

Figure 8 


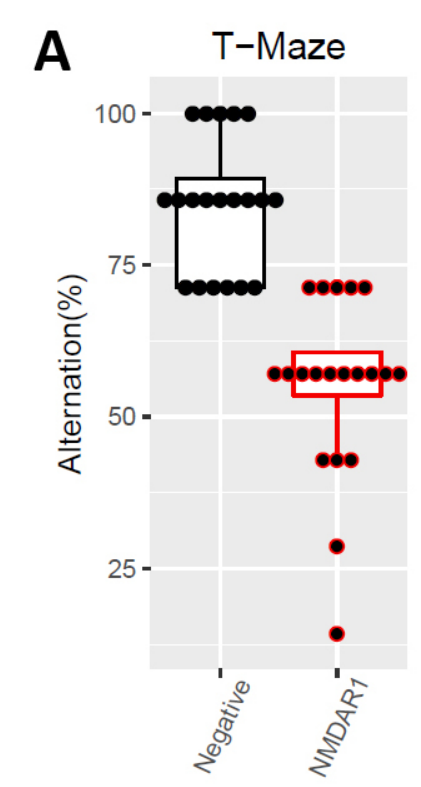

Autoantibody

D

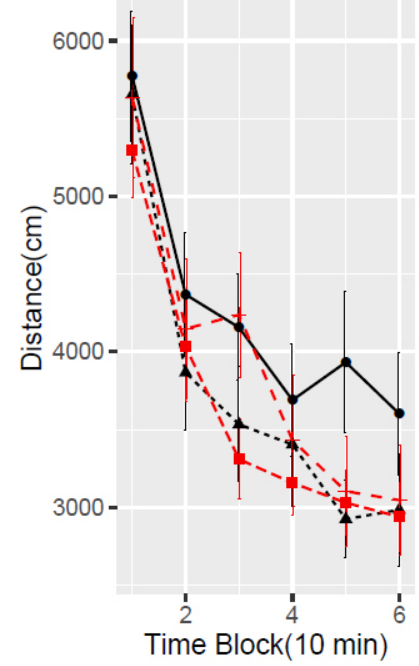

G

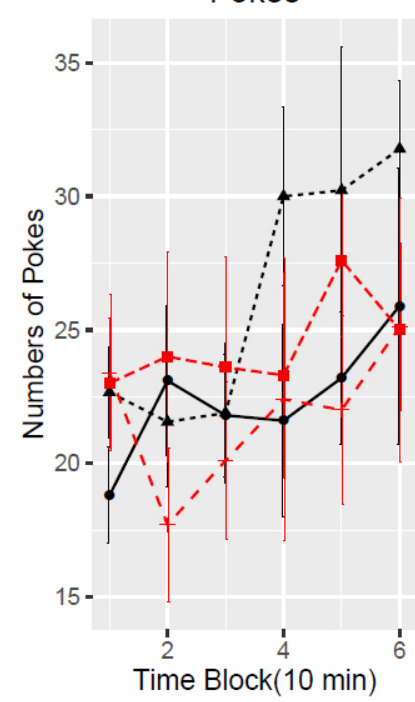

B

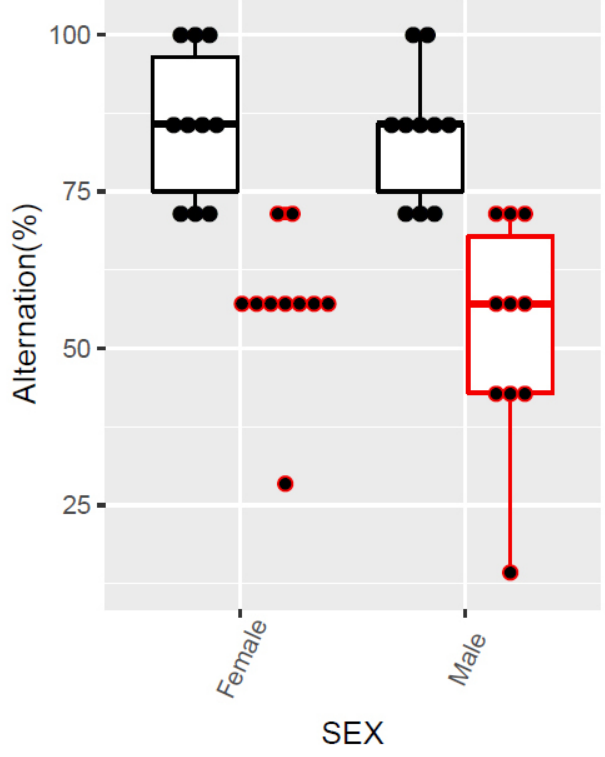

ANTIBODY

E Negative

由 NMDAR1

C

\begin{tabular}{|l|r|}
\hline T-Maze Power Analysis \\
\hline Sample size & $\begin{array}{l}\text { Negative }=20 \\
\text { NMDAR } 1=20\end{array}$ \\
\hline ANOVA F & $F(1,36)=51.3$ \\
\hline$p$ value & $2.02 E-08$ \\
\hline Effect size (d) & 2.31 \\
\hline Power & 1 \\
\hline
\end{tabular}
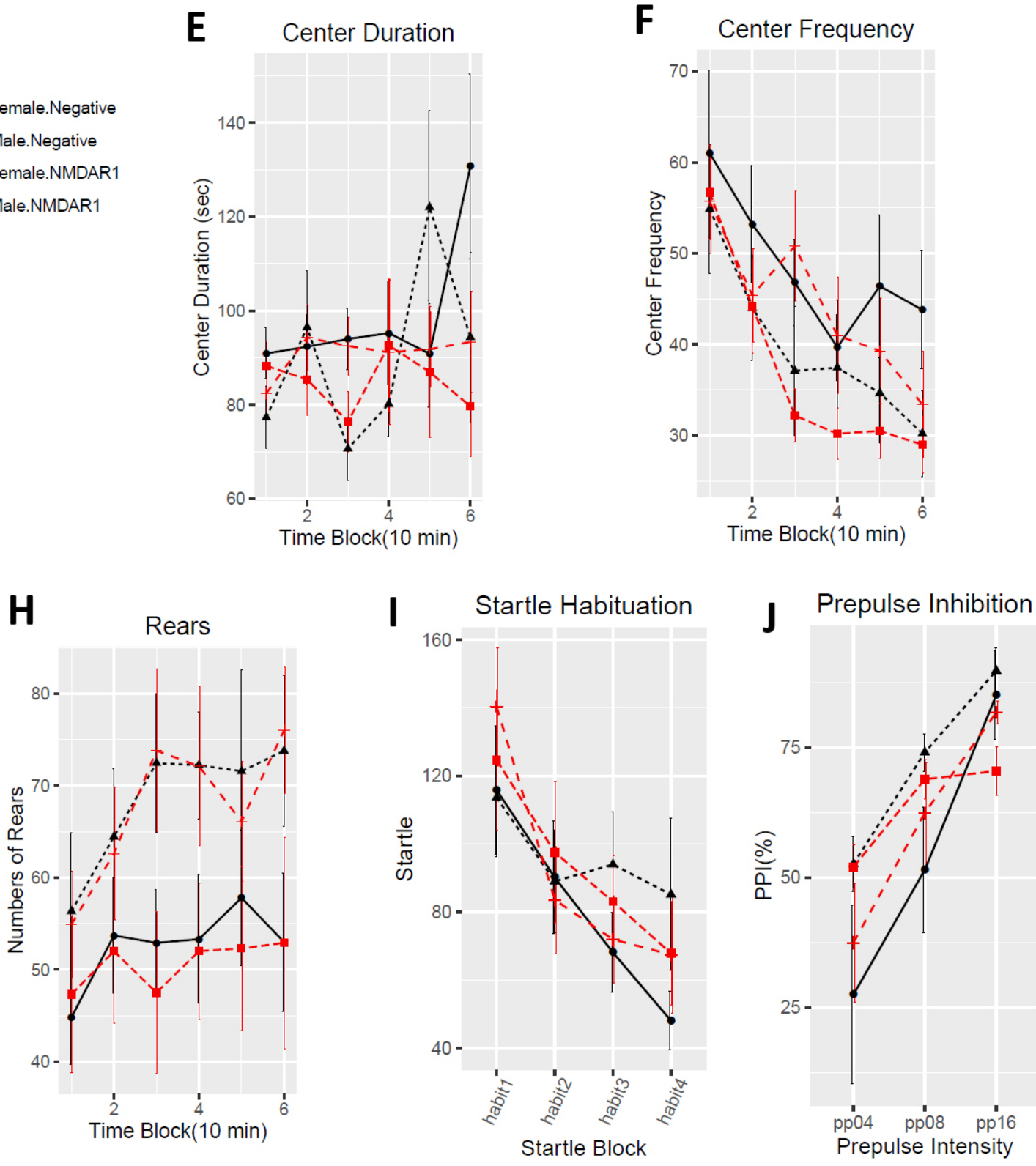

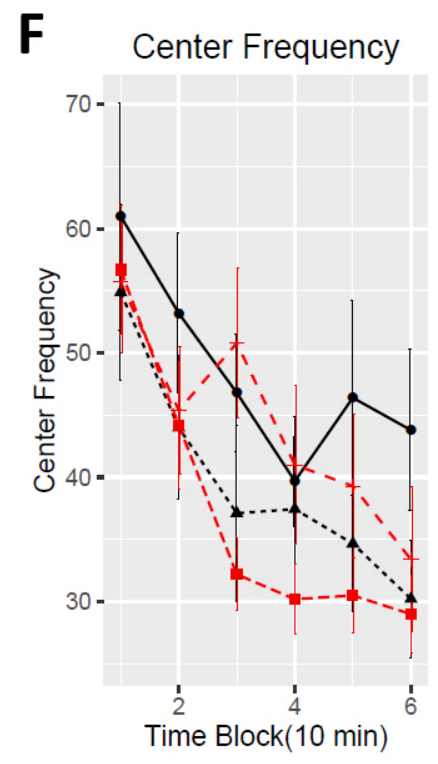

Startle Block 


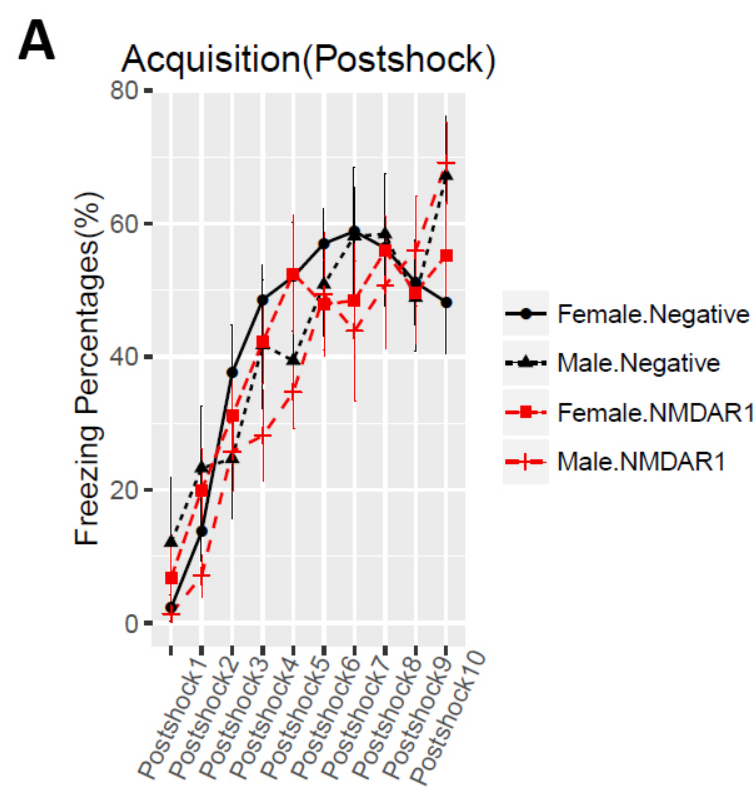

Postshock

D

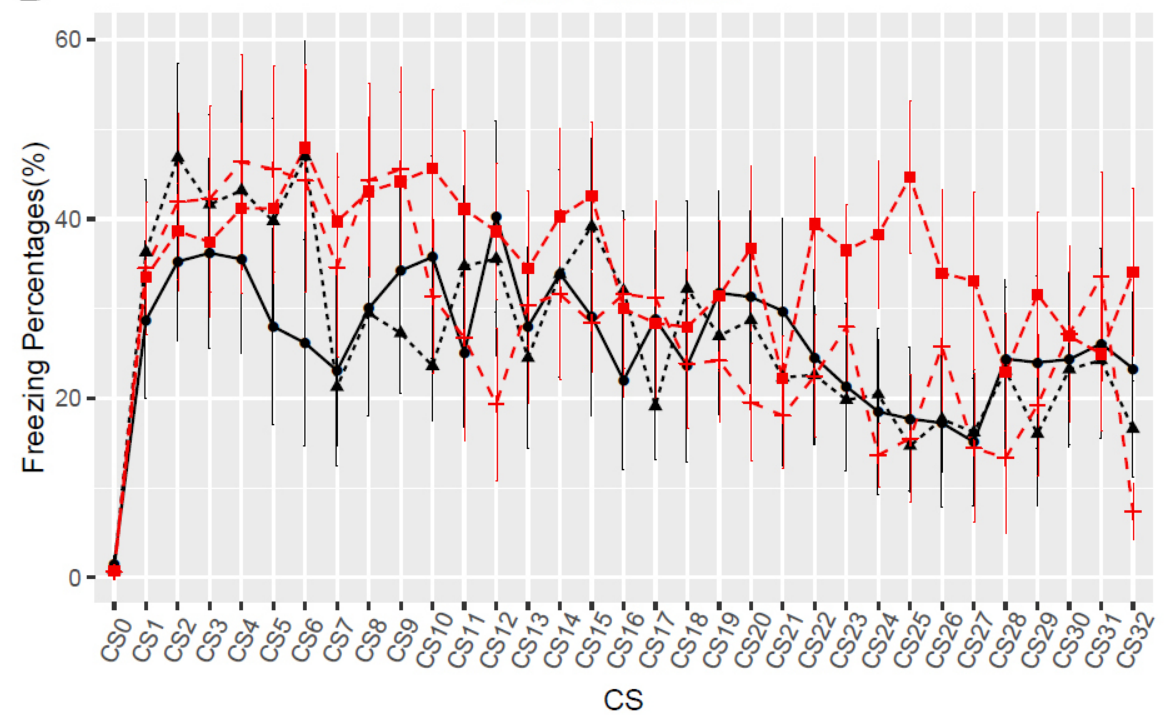

B Acquisition(CS)

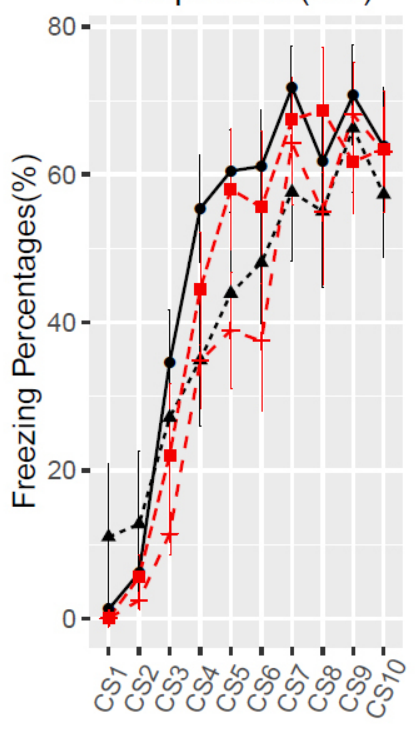

CS
C Context Memory

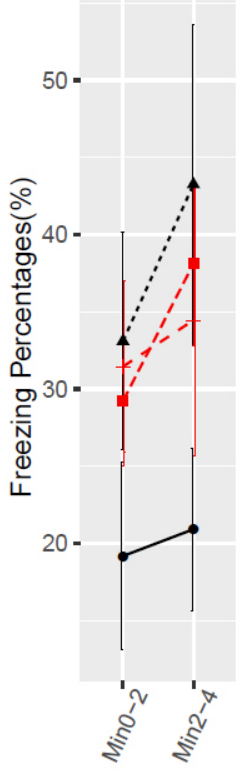

E

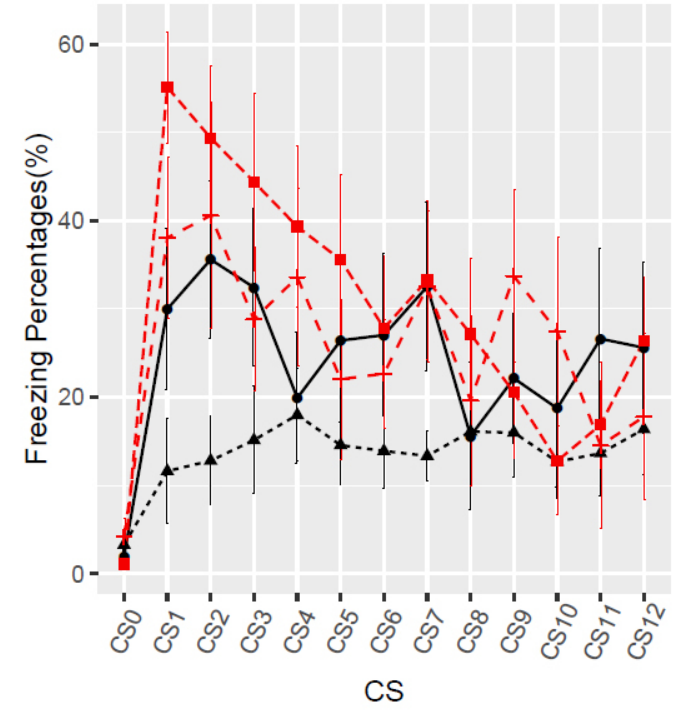

Figure 10 
$\frac{\sum}{\frac{E}{3}}$

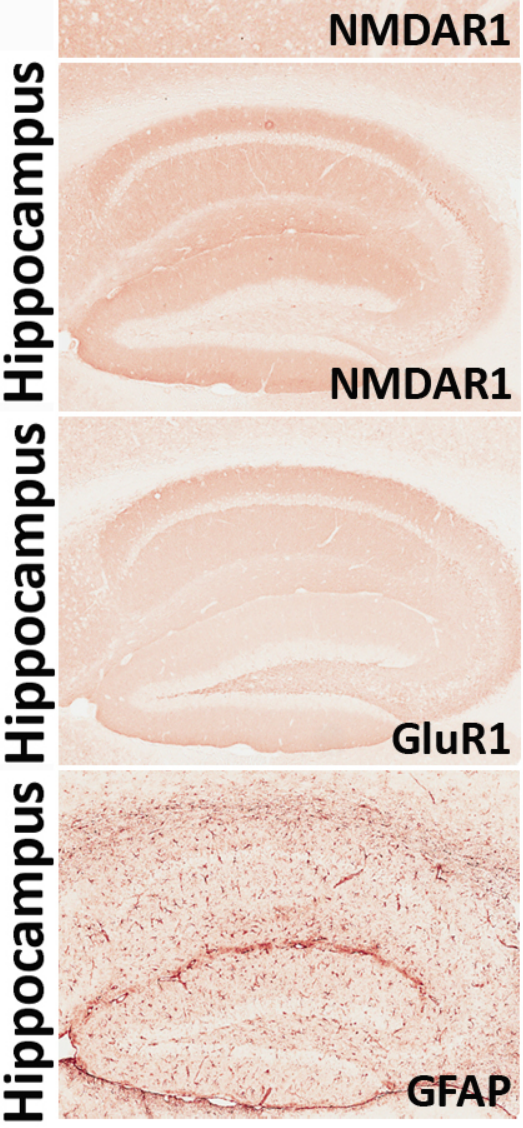

NMDAR1

NMDAR1

NMDAR1

NMDAR1

NMDAR1

NMDAR1

GluR1

GluR1

GluR1

GFAP
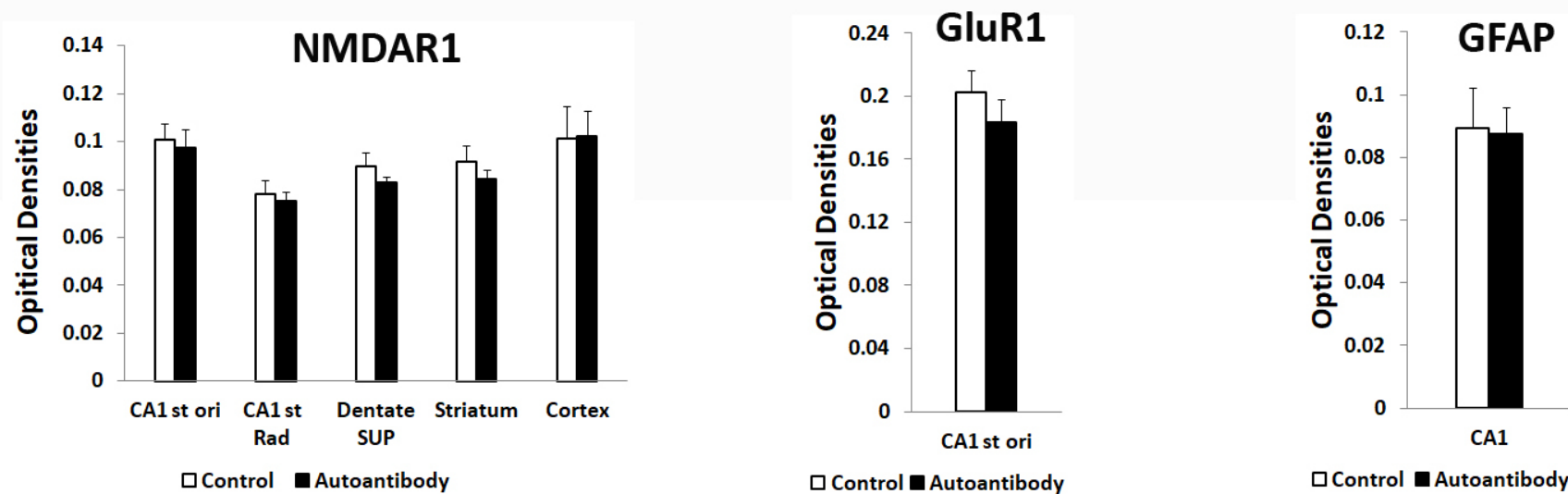

$\square$ Control $\square$ Autoantibody

$\square$ Control $\square$ Autoantibody 
TABEL I. Summary of Behavioral Analysis

\begin{tabular}{lccc}
\hline & Pilot Cohort & Large Cohort & Replication \\
\hline Mice & C: $7(\mathrm{M})$ & C: $10(\mathrm{M}), 9(\mathrm{~F})$ & C: $10(\mathrm{M}), 10(\mathrm{~F})$ \\
& P: $5(\mathrm{M})$ & P: $10(\mathrm{M}), 10(\mathrm{~F})$ & P: $10(\mathrm{M}), 10(\mathrm{~F})$ \\
\hline
\end{tabular}

\section{T-Maze}

Spontaneous Alternation

$p=0.06$

$\mathrm{p}=0.0003^{* * *}$

$\mathrm{p}=2.02 \mathrm{e}-08^{* * *}$

\section{Locomotion}

Total Distance

ns

ns

ns

Center Duration

ns

ns

ns

Center Frequency

ns

ns

ns

Pokes

ns

Rears

ns

PPI

Startle

ns

ns

ns

Prepulse Inhibition

ns

ns

ns

\section{Fear Conditioning}

Fear Acquisition

$p=0.024$

ns

ns

Context Fear

ns

ns

ns

Fear Extinction

$p=0.001$

ns

ns

Fear Extinction Recall

$p=0.019$

ns

$p=0.00056$ \#

\footnotetext{
\# Antibody X CS Interaction
} 